Accepted by Icarus on February 19, 2014

\title{
Orbit and Bulk Density of the OSIRIS-REx Target Asteroid (101955) Bennu
}

\author{
Steven R. Chesley*1, Davide Farnocchia ${ }^{1}$, Michael C. Nolan ${ }^{2}$, David Vokrouhlický ${ }^{3}$, Paul W. \\ Chodas $^{1}$, Andrea Milani ${ }^{4}$, Federica Spoto ${ }^{4}$, Benjamin Rozitis ${ }^{5}$, Lance A. M. Benner ${ }^{1}$, William F. \\ Bottke $^{6}$, Michael W. Busch ${ }^{7}$, Joshua P. Emery ${ }^{8}$, Ellen S. Howell ${ }^{2}$, Dante S. Lauretta ${ }^{9}$, Jean-Luc \\ Margot $^{10}$, and Patrick A. Taylor ${ }^{2}$
}

\begin{abstract}
The target asteroid of the OSIRIS-REx asteroid sample return mission, (101955) Bennu (formerly $1999 \mathrm{RQ}_{36}$ ), is a half-kilometer near-Earth asteroid with an extraordinarily well constrained orbit. An extensive data set of optical astrometry from 1999-2013 and high-quality radar delay measurements to Bennu in 1999, 2005, and 2011 reveal the action of the Yarkovsky effect, with a mean semimajor axis drift rate $d a / d t=(-19.0 \pm 0.1) \times 10^{-4} \mathrm{au} / \mathrm{Myr}$ or $284 \pm 1.5 \mathrm{~m} / \mathrm{yr}$. The accuracy of this result depends critically on the fidelity of the observational and dynamical model. As an example, neglecting the relativistic perturbations of the Earth during close approaches affects the orbit with $3 \sigma$ significance in $d a / d t$.

The orbital deviations from purely gravitational dynamics allow us to deduce the acceleration of the Yarkovsky effect, while the known physical characterization of Bennu allows us to independently model the force due to thermal emissions. The combination of these two analyses yields a bulk density of $\rho=1260 \pm 70 \mathrm{~kg} / \mathrm{m}^{3}$, which indicates a macroporosity in the range $40 \pm 10 \%$ for the bulk densities of likely analog meteorites,

* steve.chesley@jpl.nasa.gov

${ }^{1}$ Jet Propulsion Laboratory, California Institute of Technology, 4800 Oak Grove Drive, Pasadena, CA 91109, USA

${ }^{2}$ Arecibo Observatory, Arecibo, PR, USA

${ }^{3}$ Charles Univ., Prague, Czech Republic

${ }^{4}$ Univ. di Pisa, Pisa, Italy

${ }^{5}$ Open Univ., Milton Keynes, UK

${ }^{6}$ Southwest Research Institute, Boulder, CO, USA

${ }^{7}$ SETI Inst., Mountain View, CA, USA

${ }^{8}$ Univ. Tennessee, Knoxville, TN, USA

${ }^{9}$ Univ. Arizona, Tucson, AZ, USA

${ }^{10}$ Univ. California, Los Angeles, CA, USA
\end{abstract}


suggesting a rubble-pile internal structure. The associated mass estimate is $(7.8 \pm 0.9) \times$ $10^{10} \mathrm{~kg}$ and $G M=5.2 \pm 0.6 \mathrm{~m}^{3} / \mathrm{s}^{2}$.

Bennu's Earth close approaches are deterministic over the interval 1654-2135, beyond which the predictions are statistical in nature. In particular, the 2135 close approach is likely within the lunar distance and leads to strong scattering and therefore numerous potential impacts in subsequent years, from 2175-2196. The highest individual impact probability is $9.5 \times 10^{-5}$ in 2196 , and the cumulative impact probability is $3.7 \times 10^{-4}$, leading to a cumulative Palermo Scale of -1.70 .

Subject headings: Near-Earth objects; Orbit determination; Celestial mechanics; Yarkovsky Effect; Impact Hazard

\section{Introduction}

The Apollo asteroid (101955) Bennu, a half-kilometer near-Earth asteroid previously designated $1999 \mathrm{RQ}_{36}$, is the target of the OSIRIS-REx sample return mission. A prime objective of the mission is to measure the Yarkovsky effect on this asteroid and constrain the properties that contribute to this effect. This objective is satisfied both by direct measurement of the acceleration imparted by anisotropic emission of thermal radiation, the first results of which are reported here, and by constructing a global thermophysical model of the asteroid to confirm the underlying principles that give rise to this effect.

Bennu was discovered by the LINEAR asteroid survey in September 1999. Since then, more than 500 optical observations have been obtained for this Potentially Hazardous Asteroid (PHA). Moreover, the asteroid was observed using radar by the Arecibo and Goldstone radio telescopes during three different apparitions. Thanks to this rich observational data set, Bennu has one of the most precise orbits in the catalog of known near-Earth asteroids. The exceptional precision of the Bennu orbit allows one to push the horizon for predicting possible Earth impacts beyond the 100 years typically used for impact monitoring (Milani et al. 2005), and indeed Milani et al. (2009) showed that Earth impacts for Bennu are possible in the second half of the next century. In particular, the cumulative impact probability they found was approximately $10^{-3}$, about half of which was associated with a possible impact in 2182 . However, the occurrence of an impact depends decisively on the Yarkovsky effect because the prediction uncertainty due to this nongravitational perturbations dominates over the orbital uncertainty associated with astrometric errors.

The Yarkovsky effect is a subtle nongravitational perturbation that primarily acts as a secular variation in semimajor axis and thus causes a runoff in orbital anomaly that accumulates quadratically with time (Bottke et al. 2006). The computation of the Yarkovsky perturbation requires a rather complete physical model of the asteroid, including size, shape, density, spin rate and orien-

tation, thermal properties, and even surface roughness (Rozitis and Green 2012). Though such a complete profile is rarely available, the orbital drift due to the Yarkovsky effect can sometimes be 
determined from an asteroid observational data set. For example, Chesley et al. (2003) managed to directly estimate the Yarkovsky effect for asteroid (6489) Golevka by using three radar apparitions. Vokrouhlický et al. (2008) employ the Yarkovsky effect to match precovery observations of asteroid (152563) $1992 \mathrm{BF}$ that are incompatible with purely gravitational dynamics. More recently Nugent et al. (2012) and Farnocchia et al. (2013b) have estimated the Yarkovsky effect for a few tens of near-Earth asteroids by using a formulation that depends on a single parameter to be determined from the orbital fit.

Besides Bennu, there are two other asteroids for which possible impacts are known to be driven by the Yarkovsky effect: (29075) 1950 DA (Giorgini et al. 2002) and (99942) Apophis (Chesley 2006. Giorgini et al. 2008). The relevance of the Yarkovsky effect for Apophis is due to a scattering close approach in 2029 with minimum geocentric distance $\sim 38000 \mathrm{~km}$. For 1950 DA the influence of the Yarkovsky effect for an impact in 2880 is due to the long time interval preceding the potential impact. However, no estimate of the Yarkovsky perturbation acting on these two asteroids is currently available. To analyze such cases one can use the available physical constraints for the specific objects, along with general properties of near-Earth asteroids (e.g., albedo, thermal inertia, bulk density, etc.) to statistically model the Yarkovsky effect. The orbital predictions and the impact hazard assessment are then performed by a Monte Carlo simulation that accounts for both the Yarkovsky effect distribution and the orbital uncertainty (Farnocchia et al. 2013a; Farnocchia and Chesley 2014). For Bennu, no such heroics are required. As we shall see, we now have a precise estimate of the orbital deviations caused by the Yarkovsky effect, as well as a comprehensive physical model distilled from numerous investigations.

While the Yarkovsky effect requires a priori knowledge of several physical parameters to be computed directly, its detection through orbital deviations can be used to constrain the otherwise unknown physical parameters. When the spin state is unknown, one can derive weak constraints on obliquity, as was first shown by Vokrouhlický et al. (2008) for 1992 BF. In cases where the spin state is well characterized, usually through the combination of radar imaging and photometric light curves, the bulk density of the object is correlated with the thermal properties and mutual constraints can be inferred, as was the case for Golevka (Chesley et al. 2003). Rozitis et al. (2013) were able to jointly model the measured Yarkovsky and YORP effects on (1862) Apollo, and thereby constrain a number of the body's physical characteristics, including axis ratios, size, albedo, thermal inertia and bulk density. In the case of Bennu, the thermal inertia is known from infrared observations (Emery et al. 2014; Müller et al. 2012), and so we are able to directly estimate the mass and bulk density. 


\section{Observational Data and Treatment}

\subsection{Optical Astrometry}

We use the 569 RA-DEC astrometric measurements available from the Minor Planet Center from 1999-Sep-11.4 to 2013-Jan-20.1. We apply the star catalog debiasing algorithm introduced by Chesley et al. (2010), and data weights are generally based on the astrometric weighting scheme proposed in Sec. 6.1 of that paper. In some cases there is an excess of observations from a single

observatory in a single night. In such cases we relax the weights by a factor of about $\sqrt{N / 5}$, where $N$ is the number of observations in the night. This reduces the effect of the particular data set to a level more consistent with the typical and preferred contribution of 3-5 observations per night.

Considerable care was taken in identifying outlier observations to be deleted as discordant with the bulk of the observations. From among the 569 available observations from 43 stations, we reject 91 as outliers, leaving 478 positions from 34 stations in the fits. Figure 1 depicts the postfit plane of sky residuals, highlighting the deleted data. There are an additional 14 observations, all deleted, that are not depicted in Fig. 1 because they fall beyond the limits of the plot. The manual rejection approach often deletes an entire batch of data if it appears biased in the mean, thus some of the deleted points in Fig. 1 do not show significant residuals. On the other hand, some observations are de-weighted relative to the others, and in some cases these are not deleted, despite the raw residuals being larger than some rejected observations. In Sec. 3.4 we discuss the dependency of the ephemeris prediction on the outlier rejection approach.

[Figure 1 about here.]

\subsection{Radar Astrometry}

The time delay and Doppler shift of radar echoes from Bennu were measured in 1999, 2005 and 2011. Radar astrometry was obtained at both Arecibo and Goldstone as detailed in Table 1. The delay observations in the table correspond to the round-trip light travel time from the nominal telescope position to the center of mass of the object, and thus they are often referred to as range measurements. Doppler measurements in the table reflect the frequency shift between the transmit and receive signals due to the line-of-sight velocity of the object. The use of radar delay and Doppler measurements in asteroid orbit determination was introduced by Yeomans et al. (1992).

Delay uncertainties arise from the finite resolution of the imaging of 0.05-0.125 $\mu \mathrm{s} / \mathrm{px}$ (Nolan et al. 2013), uncertainty in the shape modeling (to determine the center of mass from the observed echo power) of 10-20 m, equivalent to about $0.1 \mu \mathrm{s}$, and systematic calibration, including uncertainties in the position of the telescope and light travel within the telescope optics. Because we have a shape model of Bennu that directly relates the individual range observations to the center of figure of the model (Nolan et al. 2013), the systematic uncertainties dominate the range uncertainty in the 
1999 and 2005 observations, and are assigned conservative values of 1.0 and $0.5 \mu$ s (respectively). In 2011, Bennu was much farther away than the previous observations, and the uncertainty of $2 \mu \mathrm{s}$ is from the pixel scale of the observations. Doppler uncertainties are taken to be $1 \mathrm{~Hz}$ at $2380 \mathrm{MHz}$, about $1 / 4$ of the total rotational Doppler width of the object, and are based on the uncertainty of estimating the position of the center of mass of the spectra.

The 2011 observations (Fig. 2 and Table 2) were of too low resolution and SNR to be useful for shape modeling and were obtained solely for improving our knowledge of the orbit of Bennu. The

$2-\mu \mathrm{s}(300 \mathrm{~m})$ resolution was chosen to be the finest resolution that would maximize the SNR of the observations by including all of the echo power from the 250 -m radius asteroid in one or two range bins. The asteroid was visible with a SNR $>3$ on each of the three observing dates at consistent delay and Doppler offsets from the a priori ephemeris used in the data taking.

The 2011 radar observations of Bennu, which enabled the results of the present paper, almost never happened. The two-million-Watt, 65,000-Volt "power brick" that supplies the electricity for the Arecibo Planetary Radar system failed in late 2010, and was finally repaired on September 15, 2011. Because of the critical schedule for Bennu observations, in a space of seven days the 16-ton unit was trucked 800 miles from Pennsylvania to Florida, shipped to Puerto Rico, trucked again, and lifted into place with a crane. The system was reconnected and recommissioned in four days, after nearly a year of down-time, just in time to perform the observations on the last possible dates of September 27-29, and just as the prime contractor managing the Arecibo Observatory was changing (on October 1), after 45 years of operation by Cornell University, so that most observatory operations were frozen for the transition.

[Figure 2 about here.]

[Table 1 about here.]

[Table 2 about here.]

\section{Orbit Determination and Dynamical Model}

We have updated the orbit determination for Bennu based on the observational data set described above. These orbital position measurements place extraordinary constraints on the orbit determination, and thus we must pay careful attention to the fidelity of force models, observation models and numerical integration. Our dynamical model includes direct solar radiation pressure and the thermal re-emission of absorbed solar radiation (i.e., the Yarkovsky effect). Besides the gravitational acceleration of the Sun, we include Newtonian perturbations by the eight planets, the Moon, Pluto and 25 selected main belt asteroids. We consider the oblateness term of the Earth's geopotential and full relativistic perturbations from the Sun, eight planets and the Moon. 
As shown by Giorgini et al. (2002), who studied the potential impact of 29075 (1950 DA) in the year 2880, other potential dynamical perturbations, such as galactic tide, solar mass loss and solar oblateness, are too slight to affect our results. This is because these small effects, which were not important for 1950 DA, will be even less significant for Bennu due to the much shorter time interval.

\subsection{Yarkovsky Effect}

The Yarkovsky effect is a key consideration when fitting an orbit for Bennu (Milani et al. 2009). This slight nongravitational acceleration arises from the anisotropic re-emission at thermal wavelengths of absorbed solar radiation (Bottke et al. 2006). The component of the thermal recoil acceleration in the transverse direction acts to steadily increase or decrease the orbital energy, leading to a drift in semimajor axis $d a / d t$ that accumulates quadratically with time in the orbital longitude of the asteroid. For a uniform, spherical asteroid on a known orbit, the drift rate depends on the physical characteristics of the asteroid according to

$$
\frac{d a}{d t} \propto \frac{\cos \gamma}{\rho D}
$$

where $\gamma$ is the obliquity of the asteroid equator with respect to its orbital plane, $\rho$ is the bulk density of the asteroid, and $D$ is the effective diameter. Additionally, $d a / d t$ depends in a nonlinear and often nonintuitive way on the asteroid rotation period $P$ and the surface material properties, namely thermal inertia $\Gamma$, infrared emissivity $\epsilon$ and Bond albedo $A$ (Vokrouhlický et al. 2000).

We have three models available to us for computing thermal accelerations on Bennu. The first, and most straightforward, is to simply apply a transverse acceleration of the form $A_{T} \times(r / 1 \mathrm{au})^{-d}$, where $A_{T}$ is an estimable parameter, $r$ is the heliocentric distance and the exponent is typically assumed as $d=2$ to match the level of absorbed solar radiation. Given an estimated value of $A_{T}$ and the assumed value of $d$, one can readily derive the time-averaged $d a / d t$ using Gauss' planetary equations (Farnocchia et al. 2013b). This approach, which we term the transverse model, is computationally fast and captures the salient aspects of the thermal recoil acceleration. Importantly, it requires no information about the physical characteristics or spin state of the asteroid, and so it can be implemented readily in cases where only astrometric information is available (e.g., Vokrouhlický

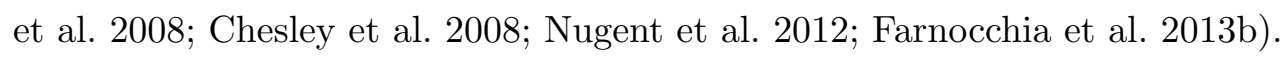

For Bennu we find numerically that the exponent $d=2.25$ provides the best match to the transverse thermal acceleration derived from the thermal re-emission models described below. This result can also be computed analytically using a simplified model with the technique described in Appendix A. Using the transverse model with $d=2.25$ we derive JPL solution 87 (Table 3), which serves as a reference solution as we investigate the effect of various model variations on the orbit.

JPL solution 87 yields a Yarkovsky drift estimate that compares well with the corresponding result from Milani et al. (2009), who used observations only through mid-2006 and found $d a / d t=$ 
$(-15 \pm 9.5) \times 10^{-4} \mathrm{au} / \mathrm{Myr}$, which was judged to be a weak detection of the nongravitational acceleration. Using the same fit span (1999-2006) from the current data set we now find $d a / d t=$ $(-22.9 \pm 5.3) \times 10^{-4} \mathrm{au} / \mathrm{Myr}$. The change in the estimate relative to that of Milani et al. (2009) is due in large part to the use of star catalog debiasing (Chesley et al. 2010), while the improved precision is due to the higher accuracy and quantity of radar delay measurements obtained through re-measurement of the 1999 and 2005 Arecibo observations, as well through as the use of tighter weights on the optical data proposed by Chesley et al. (2010). Incorporating the subsequent optical observations through 2013 leads to $d a / d t=(-21.3 \pm 4.6) \times 10^{-4}$ au/Myr. Finally, adding the 2011 Arecibo radar astrometry reduces the uncertainty by nearly a factor 50 , leading to the current best estimate $d a / d t=(-19.0 \pm 0.1) \times 10^{-4} \mathrm{au} / \mathrm{Myr}$. We note that the new formal uncertainty on $d a / d t$ is $0.5 \%$, by far the most precise Yarkovsky estimate available to date. As well, the uncertainty on the semimajor axis $a$ is $6 \mathrm{~m}$, the lowest value currently found in the asteroid catalog. This low uncertainty is primarily a reflection of the current precision of the orbital period $(2 \mathrm{~ms})$ rather than an indication of the uncertainty in the predicted asteroid position, which is at the level of a few kilometers during the fit span.

[Table 3 about here.]

Both our second (linear) and third (nonlinear) Yarkovsky acceleration models employ heat transfer models of different levels of fidelity in order to predict the surface temperature and associated re-emission of thermal energy. The linear model utilizes linearized heat transfer equations on a rotating homogeneous sphere, closely following the development given by Vokrouhlický et al. (2000) for both the diurnal and seasonal components of the Yarkovsky effect. The linear model requires knowledge of the spin orientation and rate, asteroid diameter and thermal inertia, but does not allow for shape effects such as self-shadowing and self-heating, which are generally considered minor. The linear model assumes a sphere, and so oblateness effects are not captured. This is relevant because the cross-sectional area receiving solar radiation is increased for an equal volume sphere relative to that of an oblate body, and thus the force derived with the linear model is enhanced relative to the nonlinear model. This in turn leads to an increased estimate of the bulk density as we shall see later.

The nonlinear model is the highest fidelity Yarkovsky force model that we apply to the orbit determination problem. This approach solves the nonlinear heat transfer equation on a finiteelement mesh of plates or facets that models the Nolan et al. (2013) asteroid shape. The approach is described in more detail by Čapek and Vokrouhlický (2005), but we summarize it here. For each facet on the asteroid shape model, the nonlinear heat transfer problem is solved while the asteroid rotates with a constant spin rate and orientation and revolves along a frozen, two-body heliocentric orbit. A uniform temperature distribution is assumed at start-up and the temperature and energy balance between absorbed, conducted and re-radiated radiation for each facet is solved as a function of time. The heat transfer problem is treated as one-dimensional, i.e., the temperature for a given facet depends only on the depth below the facet. There is no conduction across or between facets. 
After several orbital revolutions the temperature profile from revolution to revolution converges for each plate. Following convergence, diurnal averaging of the vector sum of the thermal emission over the body yields the force of thermal emission as a function of orbital anomaly. Given the shape model volume and an assumed bulk density, the mass can be computed and from this the thermal recoil acceleration. This ultimately leads to a lookup table of acceleration as a function of true anomaly that is interpolated during the high-fidelity orbital propagation.

The nonlinear model was previously used with asteroid (6489) Golevka (Chesley et al. 2003), but at that time the acceleration table was for a frozen orbit, which turns out to be an unacceptable approximation for Bennu. Figure 3 shows the orbital element variations into the future due to planetary perturbations and Fig. 4 reveals the associated variation in the average $d a / d t$, which is clearly significant relative to the $0.10 \times 10^{-4}$ au/Myr uncertainty. As a result of this analysis we have implemented an enhancement to the nonlinear model that corrects the tabulated accelerations for variations in orbital elements. The approach is to compute the Yarkovsky force vector from a linearized expansion about a central, reference orbit according to

$$
\vec{F}_{Y}\left(a, e ; f_{i}\right)=\vec{F}_{Y}\left(a_{0}, e_{0} ; f_{i}\right)+\frac{\partial \vec{F}_{Y}\left(a_{0}, e_{0} ; f_{i}\right)}{\partial a}\left(a-a_{0}\right)+\frac{\partial \vec{F}_{Y}\left(a_{0}, e_{0} ; f_{i}\right)}{\partial e}\left(e-e_{0}\right) .
$$

Here $\vec{F}_{Y}$ is the thermal acceleration in the orbit plane frame so that variations of the Keplerian Euler angle orbital elements $(\omega, \Omega, i)$ do not affect the computation; $\vec{F}_{Y}$ is rotated to the inertial frame during the propagation. The $f_{i}$ are the true anomaly values in the tabulation, $a_{0}$ and $e_{0}$ are the values for the reference orbit. The partial derivatives are also tabulated after they are derived by finite differences based on a series of pre-computed lookup tables for varying orbits $\vec{F}_{Y}\left(a_{0} \pm \delta a, e_{0} \pm \delta e ; f_{i}\right)$.

[Figure 3 about here.]

[Figure 4 about here.]

When computing an orbit with the linear or nonlinear model we use the physical parameters listed in Table 4, and for the nonlinear model we also use the Nolan et al. (2013) shape model. The bulk density $\rho$ is estimated as a free parameter. Because the semimajor axis drift $d a / d t$ is constrained by the observations at the $0.5 \%$ level, any variations in the force computed by the thermal models manifests as a variation in the estimated bulk density. This is discussed in greater detail in Sec. 4 .

[Table 4 about here.]

[Figure 5 about here.] 
[Figure 6 about here.]

JPL solution 85 (Table 3) uses the nonlinear model, and is assumed to be our most accurate orbital solution. To indicate the differences between the two models, Fig. 5 depicts the estimated thermal recoil accelerations from the nonlinear and linear models. The plot reveals an excellent agreement between the two models in the transverse acceleration, which is to be expected since the transverse component is constrained by the observed orbital runoff and the associated semimajor axis drift. The radial and normal (out-of-plane) accelerations show a good but imperfect agreement, with linear model accelerations being noticeably reduced relative to the nonlinear model.

Figure 6 shows how the radial and transverse accelerations in the linear model affect the instantaneous and average values of $d a / d t$, as derived from the classical Gauss planetary equations. During a given orbit, the variations in the instantaneous drift rate are much larger from the transverse component. The normal component of acceleration does not affect the semimajor axis. While the radial component of acceleration does lead to variations in semimajor axis during an orbital period, in the mean the radial term does not contribute to semimajor axis drift, which is a classical result if the radial accelerations are symmetric about perihelion. However, Fig. 5 reveals that symmetry is not necessarily present in this case. There is a slight hysteresis in the radial acceleration profile for the linear model, but because the curve crosses itself the integrated area under the curve in one orbit nets to approximately zero. In contrast, the nonlinear radial acceleration has a more significant hysteresis that does not sum to zero, and thus the radial component of acceleration actually contributes to $d a / d t$ in the mean. This behavior is presumably associated with the fact that thermal energy penetrates more deeply below the asteroid surface around perihelion when the absorbed radiation is greatest, which leads to greater thermal emission post-perihelion that pre-perihelion. We find that in the nonlinear model the radial acceleration increases $d a / d t$ by $0.3 \%$, which is not negligible relative to the $0.5 \%$ precision of the estimate. The result is that the transverse component must contribute $0.3 \%$ more in magnitude to compensate. With the linear model the radial contribution to $d a / d t$ is 60 times less.

Table 5 shows the variation in estimated $d a / d t$ associated with the different Yarkovsky models. JPL solution $87(d=2.25)$ is the reference solution for the comparison, and the linear and nonlinear models yield $d a / d t$ values within $0.03 \%$, less than a tenth of the formal uncertainty. The result for the typical default value $d=2$ is also tabulated and agrees well. This is not surprising since the astrometry provides a strong constraint on $d a / d t$ that the models must accommodate. At this level of precision, the averaged $d a / d t$ may not be the best means of quantifying the Yarkovsky effect because the mean value changes as the orbit undergoes strong planetary perturbations (Figs. 3 and 4). Nonetheless, it is informative when comparing objects and assessing the scale of the Yarkovsky effect and so we continue to use it here.

As discussed in Sec. 5, Bennu will have a close approach to Earth in 2135 at around the lunar distance. Table 5 also lists the variation in the $2135 b$-plane coordinates $\left(\xi_{2135}, \zeta_{2135}\right)$ associated with the different Yarkovsky models. We describe these coordinates more fully later, but the salient 
point is that $\Delta \zeta_{2135}$ reveals the importance of the model variation for long term predictions, while $\Delta d a / d t$ reflects the relevance to the orbital estimate over the fit span from 1999-2013.

In addition to the Yarkovsky effect, our dynamical model also includes another nongravitational perturbation related to solar radiation, namely direct solar radiation pressure (SRP, Vokrouhlický and Milani 2000). Based on the Nolan shape model and the mass estimate discussed in Sec. 4, we assume an area-to-mass ratio of $2.59 \times 10^{-6} \mathrm{~m}^{2} / \mathrm{kg}$, which leads to an acceleration of $1.2 \times 10^{-11} \mathrm{~m} / \mathrm{s}^{2}$ at $1 \mathrm{au}$, an order of magnitude greater than the radial acceleration from thermal re-emissions (see Fig. 5). Reflected radiation pressure is negligible due to the $1.7 \%$ Bond albedo of the body (Emery et al. 2014). Even though the acceleration of SRP is several times greater than that from thermal re-emission, it has little effect on the orbital predictions as it is perfectly aliased with the solar gravity. Turning SRP on and off changes the estimated semimajor axis (by $\sim 67 \sigma$ ) but leaves the mean motion unchanged. Thus there is only a minor effect on the trajectory from eliminating solar radiation pressure from the force model (by assuming an area-to-mass ratio of zero), as can be seen in Table 5 under the entry labeled Area/Mass $=0$.

[Table 5 about here.]

\subsection{Gravitational Perturbers}

The gravitational effects of the Sun, eight planets, the Moon and Pluto are based on JPL's DE424 planetary ephemeris (Folkner 2011). The use of the older DE405 planetary ephemeris (Standish 2000) leads to a modest variation in the estimated $d a / d t$ and the predicted $\zeta_{2135}$ as indicated in Table 5 .

When Bennu is near the Earth we modeled the gravitational perturbation due to Earth oblateness. Table 5 indicates the effect of varying the distance within which the oblateness model is included. We found that unless the effect was included whenever the asteroid is closer than 0.3 au there is a modest but discernible effect on the orbit determination and propagation. As a result we used a 1 au cutoff as our baseline.

Perturbing asteroids were also included in the force model. Using DE424, we developed mutually perturbed trajectories of the four largest asteroids (1 Ceres, 2 Pallas, 4 Vesta and 10 Hygeia) and designated this perturber model the CPVH small body ephemeris. We then computed the orbits for the next 12 largest main belt asteroids, each of which was perturbed only by DE424 and CPVH. The combination of these 12 additions with CPVH formed a perturber list of the 16 most massive asteroids (based on current mass estimates), and we refer to this perturber model as BIG-16. Finally, we added nine more asteroids, which were selected according to an analysis of which perturbers could most significantly influence the orbit of Bennu. The final nine asteroid ephemerides, each perturbed by DE424 and BIG-16, were combined with BIG-16 to form our final, baseline perturber set of 25 asteroids. 
Table 5 indicates the effect on the estimated value of $d a / d t$ due to changing the perturber model to either BIG-16 or CPVH. In either case the effect is small and far less than the 0.5\% formal uncertainty. Table 6 lists the assumed masses for each of the asteroid perturbers, as well as the effect of deleting each one of them from the perturber model. From this table one can see that, besides the very large contribution of 1 Ceres, 2 Pallas and 4 Vesta, only two other asteroids affect $d a / d t$ at more than $0.1 \sigma$, namely 6 Hebe and 7 Iris. Beyond CPVH, the accumulation of smaller and smaller contributions tends toward a zero mean. This is not surprising, and is a fortuitous result of the low aphelion distance $(1.36 \mathrm{au})$ of Bennu, which limits the perturbations of the main asteroid belt.

[Table 6 about here.]

\subsection{Relativity}

We used a full relativistic force model including the contribution of the Sun, the planets, and the Moon. More specifically, we used the Einstein-Infeld-Hoffman (EIH) approximation (Will 1993 Moyer 2003; Soffel et al. 2003). Table 5 shows the variations in $d a / d t$ and $\zeta_{2135}$ associated with different relativistic models. We found a $1.6 \%$ difference in $d a / d t$ with respect to the basic Sunonly Schwarzschild term (Damour et al. 1994, Sec. 4). This is only in small part due to the switch to the improved model for the Sun, as the contribution of some of the planets is not negligible. In particular, the Earth's relativistic terms are responsible for a 1.5\% $(\sim 3 \sigma)$ variation because of significant short range effects during Bennu Earth approaches in 1999 and 2005. Figure 7 shows the main relativistic terms and compares them to the Yarkovsky perturbation. Clearly, the relativistic effects of the Sun are very important, about two orders of magnitude greater than Yarkovsky, though it matters little whether the Schwarzschild or EIH approximation is used. The Earth's relativistic terms are at the same level as Yarkovsky during the Earth encounters in 1999 and 2005. At other times, Jupiter and Venus perturbations are generally more significant, although even the lunar term can briefly exceed them during close Earth encounters.

The Yarkovsky effect is primarily a transverse acceleration and thus the transverse component of the relativistic perturbations can alias as Yarkovsky if not properly modeled. Figure 8 depicts how the transverse component of Earth relativistic perturbation during the 1999 close approach is several times greater than the transverse acceleration associated with the Yarkovsky effect. Because the modeled semimajor axis drift is an integral of the two curves in Fig. 8, neglecting Earth relativity leads to significant errors.

Table 5 indicates that Earth's relativity term is the most significant factor among all of those considered, at least on longer timescales as indicated by $\Delta \zeta_{2135}$. On shorter timescales, i.e., during the fitspan, Table 6 reveals that the perturbation of Vesta leads to a greater change in $\Delta d a / d t$ than Earth relativity, although Earth relativity is still more important than Vesta for longer integrations. The uncertainty in both of these perturbations is negligible. 
[Figure 7 about here.]

[Figure 8 about here.]

\subsection{Outlier Treatment}

The selection of outliers has a statistically significant effect on the orbital prediction. To explore this sensitivity, we have generated for comparison several orbital solutions with a variety of automatic outlier rejection parameter settings. These are summarized in Table 5, which lists the $\chi_{\text {rej }}$ parameter value used in the algorithm described by Carpino et al. (2003). The outlier rejection threshold $\chi_{\text {rej }}$ is similar to the sigma level at which outlier rejection takes place, but the algorithm is more sophisticated than simple sigma clipping. The number of observations deleted in the various cases is also tabulated. The importance of careful attention to statistical outliers is indicated by the fact that the solutions are seen to progress steadily towards solution 87 as progressively more stringent requirements are placed on the outlier selection. In terms of estimated $d a / d t$, the most inclusive approach to outliers falls about $0.5 \sigma$ from the solution 87 estimate. However, even a cursory inspection of the data indicates that numerous spurious points remain in the fit for that solution. While the manual outlier rejections in solution 87 are more aggressive than even the most stringent automatic selections (e.g., $\chi_{\text {rej }}=1$ ), the separation between these two solutions is slight, and both are very well constrained by the observational data, with 478 and 519 optical observations, respectively. Most of the movement in the orbital predictions due to outlier treatment can be traced to a small handful of observatories with significantly biased observations. In general, the manual approach deletes more observations because it often removes the entire contribution from a problematic observatory, rather than only those that are clearly discordant with the bulk of the data.

\subsection{Numerics and Software Validation}

Giorgini et al. (2002) show that numerical integration errors are not significant for the case of the year 2880 potential impact of 1950 DA. We reach the same conclusion for Bennu by varying the integration error tolerance used in our software. Table 5 shows that the estimated value of $d a / d t$ is not materially affected by integrator tolerance values $\leq 10^{-14}$.

All of the results in this paper are based on the outputs of the JPL orbit determination and propagation software package. We have verified our primary JPL results by careful cross-referencing with comparable results obtained with the OrbFit orbit determination and integration packag@ ${ }^{1}$, We compared the orbital solutions, the sensitivity to different settings of the dynamical model,

\footnotetext{
${ }^{1}$ See http://adams.dm.unipi.it/orbfit; we used the OrbFit version 4.3, which is still in beta testing.
} 
and the orbit propagation, and we found that these two independent software packages reproduce each other's results very well. Indeed, the comparison with the OrbFit software package revealed to us the critical importance of the Earth general relativity terms in the dynamical model. After resolving modeling discrepancies, we found that the determinations of $A_{T}$ from the orbital fits was consistent to better than $0.1 \%$, corresponding to $<0.2 \sigma$ in $d a / d t$. We are therefore confident that our findings are not corrupted by software bugs.

\section{Mass, Bulk Density and Implications}

The linear and nonlinear Yarkovsky models both require the asteroid bulk density $\rho$, which was initially unknown. However, since all other parameters in the model are independently known, we can estimate this quantity. We used the linear model to compute JPL solution 86, with an associated bulk density estimate of $1314 \mathrm{~kg} / \mathrm{m}^{3}$. Similarly, we used the more accurate nonlinear model to obtain JPL solution 85 (Table 3), which includes an associated bulk density estimate $\rho=1181 \mathrm{~kg} / \mathrm{m}^{3}$. The discrepancy between the two models is a combination of factors, but overall implies that the linear model overestimates the transverse Yarkovsky force by about $11 \%$ and thus the estimated value of $\rho$ is increased to maintain the required mean $d a / d t$. Of particular importance is the oblateness of the Bennu shape model. This flattening leads to a diminished cross-sectional area, which tends to reduce the energy input and thereby reduce the thermal recoil acceleration in the nonlinear model. According to the theory of Vokrouhlický (1998) this should account for a 5-10\% error. Additionally, the linearization of the heat transfer problem tends to slightly increase the thermal emissions (Čapek and Vokrouhlický 2005), which readily accounts for the remaining discrepancy.

The uncertainty in the bulk density estimate is a complex story due to the numerous parameters that are used in formulating the estimate. The formal uncertainty that is obtained directly from the orbit determination (Table 3) captures only the $0.5 \%$ uncertainty in the semimajor axis drift, and does not account for the more significant sources of uncertainty outlined in Table 4 . The final column of that table indicates how the associated parameter uncertainty maps into the bulk density uncertainty, from which we conclude that the uncertainty in thermal inertia and asteroid size dominate over other error sources.

As described in Sec. 3.1, for a sphere we are sensitive to the product $\rho D$ and so the density estimate varies inversely with the asteroid size, in contrast to other density estimates that are derived from the asteroid volume. For a non-spherical shape the Yarkovsky acceleration actually depends on the quotient of the radiative cross-sectional area and the volume $A / V$, rather than $1 / D$, and yet the contribution to bulk density is still linear.

In contrast, the bulk density dependence on thermal inertia is markedly nonlinear (Fig. 9). The thermal inertia of Bennu is $\Gamma=310 \pm 70 \mathrm{~J} \mathrm{~m}^{-1} \mathrm{~s}^{-0.5} \mathrm{~K}^{-1}$ (Emery et al. 2014). This value is derived from analysis of a suite of observations of thermal flux, consisting of $8-20 \mu \mathrm{m}$ spectra of opposite 
hemispheres and photometry at 3.6, 4.5, 5.8, 8.0, 16, and $22 \mu \mathrm{m}$ of 10 different longitudes using the Spitzer Space Telescope. The thermophysical modeling that results in this thermal inertia estimate incorporates the detailed shape and spin information derived from radar imaging and visible light curve photometry, and explicitly includes the effects of macroscopic surface roughness.

[Figure 9 about here.]

Previous estimates of Bennu's thermal inertia (Emery et al. 2010, 2012; Müller et al. 2012) are somewhat higher $\left(\sim 600 \mathrm{~J} \mathrm{~m}^{-1} \mathrm{~s}^{-0.5} \mathrm{~K}^{-1}\right)$, and have led to correspondingly lower bulk density estimates (e.g., $970 \mathrm{~kg} / \mathrm{m}^{3}$, Chesley et al. 2012). There are two primary reasons for the different thermal inertia values. First, the earlier studies used only a subset of the Spitzer data, namely the spectra. Those spectra are noisy, making it difficult to scale the different segments of the full spectra relative to each other. Different scale values affect the best-fit model surface temperature distribution, and therefore the derived thermal inertia. In contrast, Emery et al. (2014) include the large set of photometric data, which have much higher signal-to-noise than the spectral data, leading to results that are both more accurate and have significantly smaller uncertainties. Note that if the uncertainties in scale factors are included in the uncertainty estimates from the spectral data, the error bars overlap the Emery et al. (2014) estimate given above. Second, the earlier estimates assumed a spherical shape for Bennu. However, Bennu is actually fairly oblate (Nolan et al. 2013). The oblateness causes surface facets to be tilted farther away from the Sun as compared to a sphere. The models assuming spherical shape compensate for the more direct viewing geometry with a lower thermal inertia. For these reasons, we rely here on the updated thermal inertia from Emery et al. (2014).

The effects of surface roughness on Bennu are not incorporated into the $\rho$ estimates so far, and yet Rozitis and Green (2012) used a sophisticated thermophysical model to show that the thermal effects of surface roughness always tend to increase the Yarkovsky effect. For the Bennu shape model with the roughest surface model the increase in $\rho$ is $12.7 \%$ at the nominal thermal inertia (see Fig. 10), pointing to roughness as the dominant source of uncertainty.

Although we have no rigorous estimates of Bennu's roughness, it is unlikely to be either remarkably smooth or extremely rough. The thermal inertia from Emery et al. (2014) is somewhat lower than that derived for (25143) Itokawa (Müller et al. 2005), which suggests that Bennu could have a somewhat smoother surface texture than Itokawa. Elsewhere, Nolan et al. (2013) find that the radar circular polarization ratio, which is a proxy for near-surface roughness at the scale of the radar wavelength $(12.6$ and $3.5 \mathrm{~cm})$, indicates a relatively smooth surface compared to other bodies that are not particularly rough. In particular, they find Bennu has significantly lower polarization ratios than Itokawa at both wavelengths and conclude that Bennu is likely smoother than Itokawa. However, Nolan et al. (2013) did identify a boulder on Bennu with a size of 10-20 m, suggesting the presence of smaller boulders below the resolution limit of $7.5 \mathrm{~m}$ and a surface that is not perfectly smooth. In the absence of reliable estimates, we assume that the roughness is $50 \pm 17 \%$, which 
covers the full range $0-100 \%$ at $3 \sigma$. This yields the "Enhanced" curve in Fig. 9. Inflating the reference value $\rho=1181 \mathrm{~kg} / \mathrm{m}^{3}$ by $50 \%$ of the $12.7 \%$ enhancement yields our best estimate of 1255 $\mathrm{kg} / \mathrm{m}^{3}$.

[Figure 10 about here.]

To develop a comprehensive estimate of the uncertainty in the presence of the nonlinearity evident in Fig. 9 we take a Monte Carlo approach. We sample $\Gamma, A / V$ and $\gamma$ according to the normal distributions given by Table 4. We obtain the Yarkovsky enhancement for each case by sampling a roughness from $50 \pm 17 \%$ and using it to scale the 100\%-rough enhancement (from Fig. 10) at the sampled thermal inertia. This leads to our final bulk density estimate of $\rho=1260 \pm 70 \mathrm{~kg} / \mathrm{m}^{3}$. The associated mass, GM and area-to-mass ratio values are listed in Table 7.

[Table 7 about here.]

We assume that the Yarkovsky effect is the only significant source of nongravitational acceleration on Bennu, and in particular we do not account for the possibility of outgassing, which would corrupt our bulk density estimate if it were significant. If the direction of any hypothetical outgassing is skewed towards the evening terminator, which might be expected as the diurnal thermal wave penetrates to release buried volatiles, then it would combine to increase the magnitude of the transverse acceleration on the asteroid. In this case, the bulk density estimate should be increased to account for outgassing. Conversely, if the outgassing tends to cancel the transverse thermal recoil acceleration then our bulk density estimate will be an overestimate.

3200 Phaethon, a B-type asteroid like Bennu, has long been identified as the parent body of the Geminid meteor shower (Whipple 1983). This suggests that at least some objects of this taxonomic type have the possibility of shedding material, possibly as fine-grained material entrained in gasses released by the sublimation of volatiles. However, the only report of possible dust release on Phaethon took place at a heliocentric distance of $0.14 \mathrm{au}$, and it has been suggested that the Geminids are shed from Phaethon due to fracturing associated with fatigue from thermal cycling and the decomposition of hydrated minerals (Jewitt and Li 2010). There is no evidence that Bennu is shedding material and, with $q \simeq 0.9 \mathrm{au}$, solar heating is markedly lower than that experienced by Phaethon. Therefore, we do not consider it likely that outgassing is significantly affecting our results.

Is the estimated GM in Table 7 consistent with loose material on the equator being gravitationally bound to the surface? Nolan et al. (2013) report that the maximum equatorial diameter is $565 \mathrm{~m}$. From this and the known spin rate we find a lower bound of $G M=3.7 \mathrm{~m}^{3} / \mathrm{s}^{2}$ if we assume that the gravitational attraction exceeds the centrifugal acceleration. This is a reasonable expectation because, as discussed above, we consider it likely that Bennu's surface is dominated by cm-scale and smaller regolith. However, it is difficult to rule out the possibility that some regions 
along the equator are devoid of loose material, or that induration or cohesion provides sufficient binding to keep material on the surface that would otherwise depart. Even so, the assumption that material is gravitationally bound to the surface would imply $\rho>890 \mathrm{~kg} / \mathrm{m}^{3}$ which is satisfied here with a high degree of confidence.

One can compute the macroporosity, $P=1-\rho / \rho_{M}$, of an asteroid if the bulk densities of the body $\rho$ and the appropriate meteorite analog $\rho_{M}$ are known. Bennu has been identified as a B-type asteroid, and CI and CM carbonaceous chondrite meteorite samples provide the best spectral match (Clark et al.2011). Consolmagno et al. (2008) report that CM meteorite samples have average bulk densities of $2130 \pm 190 \mathrm{~kg} / \mathrm{m}^{3}$, which, taken together with our asteroid bulk density estimate and uncertainty from Table 7, suggests $P$ in the range 30-50\%. For CI meteorites the data are fewer and less conclusive, with different measurement techniques leading to sample bulk densities similar to those of $\mathrm{CM}$ meteorites or as low as $1600 \mathrm{~kg} / \mathrm{m}^{3}$, which would allow $P$ to be as low as $20 \%$ (Consolmagno et al. 2008). Overall, our judgement is that the macroporosity of Bennu is likely to be in the range $40 \pm 10 \%$, but could be as low as $20 \%$.

Bennu's estimated bulk density is comparable to values obtained for other low-albedo asteroids, from large asteroids in the main asteroid belt to smaller asteroids in the inner solar system. The average C-type asteroid in the main belt, according to estimates derived from the gravitational perturbations on the planets, predominantly Mars, is $\rho=1290 \pm 60 \mathrm{~kg} / \mathrm{m}^{3}$ (Standish 2000). This estimate is biased toward asteroids with diameters much larger than $100 \mathrm{~km}$, which contain the majority of the mass among C-types. However, a flyby of the 53-km, C-type asteroid (253) Mathilde by the NEAR spacecraft also yielded a similar density $\rho=1300 \pm 200 \mathrm{~kg} / \mathrm{m}^{3}$ (Yeomans et al. 1997). Furthermore, Marchis et al. (2008a b) report densities of several C-complex binary asteroids in the main belt. The summary given by Marchis et al. (2008a, Table 8) suggests that the density distribution of large, C-complex binaries is $\rho=1100 \pm 300 \mathrm{~kg} / \mathrm{m}^{3}$. Among the near-Earth asteroid population, Shepard et al. (2006) report that the low-albedo binary system (276049) $2002 \mathrm{CE}_{26}$ has a $3.5-\mathrm{km}$ primary with bulk density $900_{-400}^{+500} \mathrm{~kg} / \mathrm{m}^{3}$, which is comparable within the error bars to that of Bennu.

What do Bennu's density and porosity tell us? To say anything useful here, we need to put Bennu into context. Our best estimates suggest Bennu is a fragment of a larger body that experienced a collision (Campins et al. 2010; Walsh et al. 2013). Similarly, the large multiple systems examined by Marchis et al. (2008a b) were presumably formed by large collision events (e.g., Durda et al. 2004). One would expect these smashed up target worlds, with porosity added by the fragmentation and ejecta re-assembly process, to have low bulk densities in comparison to their meteorite analogs, and that the smallest bodies should tend to still lower density due to selfgravitation and compaction on the large bodies (Baer and Chesley 2008; Baer et al. 2011). However, this size dependence seems to vanish at sizes below roughly 250-300 km, below which no obvious size trend exists in macroporosity, an observation reinforced by Bennu. With this background, we argue that Bennu's porosity was produced by a similar mechanism, consistent with our hypothesis that void space and porosity were added into Bennu by its formation and/or by post-formation 
processes. Taken together, these arguments allow us to infer that Bennu has a heavily fractured or shattered internal structure combined with a substantial porosity. These characteristics fit the definition of a rubble pile asteroid provided by Richardson et al. (2002).

\section{Earth Close Approaches}

The deterministic prediction interval for the trajectory of Bennu extends for 481 years, from 1654 to 2135. Earth close approaches within 0.05 au during this time interval are listed in Table 8 . Close encounters outside of this interval have encounter time uncertainties well in excess of a day. The closest approach in this interval is the nominally sub-lunar distance encounter in 2135 . This deep close approach leads to strong scattering of nearby orbits, and so the subsequent impact hazard can only be explored through statistical means.

Figure 3 shows the time history of Bennu's orbital elements from 2000 to 2136. There are variations of a few percent due to Earth close approaches, especially in 2135. As the Yarkovsky induced orbital drift depends on the osculating orbital elements (Farnocchia et al. 2013b), there are also commensurable variations in the $d a / d t$ evolution (see Fig. 4).

Table 5 details the effect of various differing models on the $b$-plane coordinates $\left(\xi_{2135}, \zeta_{2135}\right)$ of the close approach at the last reliably predicted Earth encounter for Bennu, which takes place in 2135. The $b$-plane is oriented normal to the inbound hyperbolic approach asymptote and is frequently used in encounter analysis. The $(\xi, \zeta)$ coordinates on the $b$-plane are oriented such that the projected heliocentric velocity of the planet is coincident with the $-\zeta$-axis. In this frame the $\zeta$ coordinate indicates how much the asteroid is early $(\zeta<0)$ or late $(\zeta>0)$ for the minimum possible distance encounter. In absolute value, the $\xi$ coordinate reveals the so-called Minimum Orbital Intersection Distance (MOID), which is the minimum possible encounter distance that the asteroid can attain assuming only changes to the timing of the asteroid encounter. For a more extensive discussion of these coordinates see Valsecchi et al. (2003) and references therein. In Table 5, the tabulated $d a / d t$ differences are indicative of the importance of the effect on the 19992012 time frame of the observation set, while $\zeta_{2135}$ provides an indication of how important the term is for the much longer integration from 2011 to 2135.

[Table 8 about here.]

\subsection{Impact Hazard Assessment}

The geometry of Bennu's orbit allows deep close approaches to the Earth, which require a careful assessment of the associated potential collision hazard. Figure 11] shows the dependence on time of the Minimum Orbit Intersection Distance (MOID, see, e.g., Gronchi 2005). According to the secular evolution, the MOID reaches its minimum near the end of the next century while short 
periodic perturbations make it cross the Earth impact cross section threshold at different epochs from 2100 to 2300 , which is therefore the time period for which we must analyze possible close approaches. This objective is similar to that discussed by Milani et al. (2009), however we bring new analysis tools to bear on the problem and we have the benefit of crucial astrometric data not available in 2009. We recall that Milani et al. (2009) based much of their analysis on the variability of the 2080 encounter circumstances, finding that, for the observational data then available, this was the last encounter that was well constrained, and after which chaotic scattering made linear analysis infeasible. With the current data set, future encounter uncertainties remain modest until after 2135 (Table 8), and nonlinear analysis techniques are necessary for subsequent encounters. Thus the 2135 encounter is the central focus in our current impact hazard assessment.

We performed a Monte Carlo sampling (Chodas and Yeomans 1999) in the 7-dimensional space of initial conditions and bulk density. Figure 12 shows the distribution of the Monte Carlo samples on the $2135 b$-plane. The $b$-plane plot depicts the geocentric locations of the incoming hyperbolic asymptote of the Monte Carlo samples on the plane orthogonal to the asymptote, indicating the distance and direction of the closest approach point of a fictitious unperturbed trajectory (see, e.g., Valsecchi et al. 2003). The linear mapping of the uncertainty region is a poor approximation as we can see from the asymmetry of the distribution. As expected, the uncertainty region gets stretched along $\zeta$, which reflects time of arrival variation and is thus related to the along-track direction.

By propagating the Monte Carlo samples through year 2250 we can determine the Virtual Impactors (VIs), i.e., the Virtual Asteroids (VAs) compatible with the orbital uncertainty corresponding to an impacting trajectory. The positions of the VIs in the $2135 b$-plane define the 2135 keyholes, which are the coordinates on the $b$-plane corresponding to a subsequent impact (Chodas 1999). On the $b$-plane of a given post-2135 encounter we can interpolate among nearby samples to identify the minimum possible future encounter distance. When this minimum distance is smaller than the Earth radius, the keyhole width is obtained by mapping the chord corresponding to the intersection between the line of variations and the impact cross section back to the $2135 b$-plane. This procedure allows us to develop a map of the keyholes in the $b$-plane. For Bennu we found about 200 keyholes in the 2135 b-plane with widths ranging from $1.6 \mathrm{~m}$ to $54 \mathrm{~km}$.

Figure 13 shows the probability density function (PDF) of $\zeta_{2135}$ resulting from the Monte Carlo sampling. As already noted, the linear approximation is not valid in this case, and so the PDF is distinctly non-gaussian. The figure also reveals the keyhole map in $\zeta_{2135}$, where the vertical bars correspond to the keyholes $>100 \mathrm{~m}$ in width and the height of the bars is proportional to the width. For a given keyhole the impact probability (IP) is simply the product of the PDF and the keyhole width. For each of the 78 keyholes larger than $100 \mathrm{~m}$ and with an IP $>10^{-10}$, Table 9 reports the impact year, the keyhole width, the impact probability, and the associated Palermo Scale (Chesley et al. 2002). The cumulative IP is $3.7 \times 10^{-4}$ and the cumulative Palermo Scale is -1.70. There are eight keyholes corresponding to an IP larger than $10^{-5}$. Among these, the year 2196 has the highest IP, $1.3 \times 10^{-4}$, which arises primarily from two separate but nearby keyholes. 
Figure 14 shows the dependence of the number of keyholes and the cumulative IP on the minimum keyhole width. Although the number of keyholes increases with decreasing minimum width, the cumulative IP is essentially captured already by only the largest $\sim 10 \%$ of keyholes, i.e., those with width $\gtrsim 1 \mathrm{~km}$.

Post-2135 Earth encounters correspond to resonant returns (Valsecchi et al. 2003). Table 10 describes the main features of the resonant returns corresponding to an IP $>10^{-5}$.

It is important to assess the reliability of our results. On one hand, the keyholes are essentially a geometric factor that does not depend on the modeling of Bennu's orbit. On the other hand, the PDF on the $2135 b$-plane can be strongly affected by the dynamical model and the statistical treatment applied to the observations. Table 5 reports the $2135 b$-plane coordinates as a function of the different configurations of the dynamical model and different settings for the removal of outliers from the observational data set. It is worth pointing out that neglecting the Earth relativistic term produces a large error comparable to a $3 \sigma$ shift in the orbital solution. In contrast, the contribution of solar radiation pressure is rather small. As already discussed in Sec. 3.1, this can be explained by the fact that the action of solar radiation pressure is aliased with the solar gravitational acceleration, and neglecting solar radiation pressure in the model is therefore compensated when fitting the orbital solution to the observations. The different Yarkovsky models give $\zeta_{2135}$ predictions within several thousand kilometers of each other. Interestingly, the shift due to the different astrometric outlier treatment is comparable to the one due to the relativistic term of the Earth and much larger than any shift due to the other dynamical configurations. Table 6 shows the effect of removing each of the 24 perturbing asteroids included in the dynamical model. Ceres, Pallas, and Vesta give the largest contributions. Among smaller perturbers, Hebe and Iris turn out to be the most important.

We used OrbFit (see Sec. 3.5) to cross-check the keyhole locations and widths, the PDF of Fig. 13, and the sensitivity to the different configurations of the dynamical model. We found good overall agreement with only one noticeable difference related to the PDF: while the PDF shapes are similar, the peaks are separated by about $40000 \mathrm{~km}$ in $\zeta_{2135}$. This difference is related to the $0.2 \sigma$ shift in the nominal solution (see Sec. 3.5 ) and is in part due to the fact that OrbFit presently uses JPL's DE405 planetary ephemeris rather than DE424, which is used in our analysis.

[Table 9 about here.]

[Table 10 about here.]

[Figure 11 about here.]

[Figure 12 about here.]

[Figure 13 about here.]

[Figure 14 about here.] 


\subsection{Statistical Close Approach Frequency}

We now want to characterize the Earth encounter history for Bennu's orbital geometry. The first step is to understand the statistical properties of Earth encounters during a node crossing cycle (see Fig. 11). For this we generated a dense sampling of 20,000 Virtual Asteroids on the Solution 87 orbit (Table 3), but with a uniform sampling of the mean anomaly from the full range, 0 to $2 \pi$, to randomize the node crossing trajectory. For each VA we recorded all of the close approaches within 0.015 au during JPL's DE424 ephemerides time interval, i.e., from year -3000 to year 3000, which contains only one node crossing cycle.

We modeled the number of Earth approaches within a given distance in a given time frame as a Poisson random variable. We estimated the Poisson parameter $\lambda$ by averaging over the trajectories of the VAs. Figure 15 shows the probability of having at least one close approach within a given geocentric distance during a node crossing cycle (dashed line). For instance, during each node crossing cycle we have $38 \%$ probability of a close approach within the lunar distance and a $6 \times$ $10^{-4}$ probability of an impact. This is consistent with our predictions for the next node crossing taking place around 2200, for which we have similar probabilities of impact and sub-lunar distance encounters.

To analyze the long-term history we need to account for the secular evolution of Bennu's orbit. As reported by NEODyS2 Bennu's perihelion precession period is $28100 \mathrm{yr}$ and each precession period contains four node crossings. For a given time interval we can compute the expected number of node crossings and suitably scale the probability of an encounter within a given distance during a single node crossing cycle. The solid lines in Fig. 15 show the probability of at least one Earth encounter within a given distance for time intervals of $1 \mathrm{yr}, 1000 \mathrm{yr}$, and 1,000,000 yr. For example, in a $1000 \mathrm{yr}$ time interval the probability of a close encounter within a lunar distance is $7 \%$ while the probability of an impact is $9 \times 10^{-5}$. This indicates that for Bennu's current orbital configuration the mean Earth impact interval is $\sim 10 \mathrm{Myr}$. Note that the precession period assumed here is for the nominal orbit of Table 3 , while the precession period does change due to planetary interactions. For instance, the nominal semimajor axis increases and the uncertainty grows after the 2135 encounter, causing the post-2135 precession period to be in the range 28900-33400 years. Delbò and Michel (2011) analyze the orbital evolution of Bennu on a much longer time frame than a single node crossing and find that the median lifetime of Bennu could be $\sim 34 \mathrm{Myr}$, but their study allowed for substantial orbital evolution to take place, while our results are valid for the present-day, un-evolved orbit.

[Figure 15 about here.]

\footnotetext{
${ }^{2}$ http://newton.dm.unipi.it/neodys/index.php?pc=1.1.6\&n=bennu
} 


\section{OSIRIS-REx Science}

Continued study of Bennu's trajectory is a significant element of the OSIRIS-REx science investigation. In particular, the characterization of the Yarkovsky effect is planned to be conducted on two tracks. On one track, Earth-based radio tracking of the spacecraft and optical navigation images of the asteroid from the spacecraft will be used to derive high-precision asteroid position measurements. These position updates will afford refined estimates of the nongravitational accelerations that the asteroid experiences. On the other track, science observations by the OSIRIS-REx spacecraft will allow the development of a complete thermophysical model of the asteroid, yielding a precise estimate of the thermal recoil acceleration, as well as direct and reflected solar radiation pressure acting on the body. A comparison of the acceleration profile from these two independent approaches will provide significant insight into the quality of current thermophysical models, and, for example, the extent to which surface roughness affects the net thermal recoil acceleration Rozitis and Green 2012).

But first the OSIRIS-REx must rendezvous with Bennu, and knowledge of the asteroid position is required for accurate navigation of the spacecraft during the initial encounter. Our current prediction calls for Radial-Transverse-Normal (RTN) position uncertainties of $(3.3,3.8,6.9) \mathrm{km}$ on 2018-Sep-10, during the planned OSIRIS-REx rendezvous. These are formal 1-sigma error bars, and may not account for some unmodelled systematic effects, although we are not aware of any that are significant. In any case, such low uncertainties suggest that asteroid ephemeris errors will not be a significant complicating factor during the OSIRIS-REx rendezvous with Bennu.

To characterize the Bennu ephemeris improvement provided by the OSIRIS-REx mission, we simulate 8 post-rendezvous, pseudo-range points from the geocenter to the asteroid center of mass. The simulated measurements are placed at monthly intervals from 2018-Dec-01 to 2019-Jul-01, and they assume an a priori uncertainty of $0.1 \mu \mathrm{s}$ in time delay, which translates to $15 \mathrm{~m}$ in range. The trajectory constraints from the OSIRIS-REx radio science effort are likely to be somewhat better than assumed for this study. Table 11 lists the uncertainties obtained before and after the inclusion of these simulated OSIRIS-REx radio science data. We find that the uncertainty in the transverse nongravitational acceleration parameter $A_{T}$, and by extension the uncertainty in the mean $d a / d t$, drops by roughly a factor $6-7$, bringing the precision to better than $0.1 \%$.

The OSIRIS-REx radio science observations will not only refine the Yarkovsky acceleration acting on the asteroid, but also enable significantly improved future predictions. Table 11 reveals that our current predictions call for position uncertainties of a few $\mathrm{km}$ at the end of proximity operations on 2020-Jan-04, which could be reduced to under $100 \mathrm{~m}$ with the simulated mission

data. The associated velocity uncertainties are of order $1 \mathrm{~mm} / \mathrm{s}$ with current information, but could fall by a factor 50 or more with the OSIRIS-REx data.

Similarly, we find that the OSIRIS-REx radio science data could narrow the $\zeta_{2135}$ uncertainty region on the $2135 b$-plane by a factor $\sim 60$. This would be similar to the reduction in uncertainty seen between the Milani et al. (2009) paper and the present paper. The implication is that the 
hazard assessment will be dramatically altered by the OSIRIS-REx radio science effort. The selfsimilar nature of the keyholes on the $2135 b$-plane suggest that the cumulative probability is likely to remain around $10^{-4}$, although if the nominal $\zeta_{2135}$ prediction does not change appreciably the cluster of relatively wide keyholes near the current nominal (Fig. 13) could lead to a cumulative probability of impact in excess of $10^{-2}$.

Besides providing direct radio science position measurements of the asteroid, OSIRIS-REx will refine and test other aspects of the Bennu ephemeris problem. The mission objectives include

- a search for outgassing and the incorporation of any activity into force models,

- direct measurement of the asteroid mass, providing ground truth for the mass determination technique presented here,

- precision radiometry of both reflected and thermally emitted radiation with high spatial resolution, providing ground truth for the thermal accelerations presented in this paper, and

- analysis of the returned sample, which will provide a direct measurement of the thermal, dielectric, and bulk density of the asteroid surface.

\section{[Table 11 about here.]}

\section{Discussion and Conclusions}

Understanding of an asteroid's physical properties becomes essential whenever the Yarkovsky effect or other nongravitational accelerations are a crucial aspect of the orbit estimation problem. Radar astrometry of asteroids can provide surprising and important constraints, not only on an asteroid's orbit, but also on its physical properties. In the case of Bennu, this information has immense value for space mission designers. We have seen that the availability of well-distributed radar astrometry over time spans of order a decade can constrain asteroid orbits to the extent that precise estimates of the Yarkovsky effect can be derived. When coupled with thermal inertia information derived from other sources, such as the Spitzer Space Telescope, important parameters such as mass, bulk density and porosity can be derived. Combining Yarkovsky detections with thermal inertia measurements to infer the asteroid mass can be implemented on other near-Earth asteroids, including potential space mission targets. This technique is the focus of ongoing work. Indeed, Bennu clearly demonstrates that even weak radar detections can have considerable science value, raising the imperative to aggressively pursue every available radar ranging opportunity for potential Yarkovsky candidates.

Our bulk density estimate for Bennu implies a primitive body with high porosity of $40 \pm 10 \%$. The implication is that Bennu must be comprised of a gravitationally bound aggregate of rubble, a conclusion that is reinforced by its shape, which is spheroidal with an equatorial bulge consistent 
with the downslope movement and accumulation of loose material at the potential minimum found at the equator (Nolan et al. 2013). This bodes well for the OSIRIS-REx sample collection effort, which requires loose material at the surface for a successful sample collection, although nothing in this study constrains the size distribution of the surface material.

The statistical encounter frequency with Earth (Fig. 15) can be used to understand the rate of encounters that could alter the shape and spin state of a body through tidal interactions (e.g., Walsh and Richardson 2008; Nesvorný et al. 2010). Scheeres et al. (2005) have shown that tidal interactions at a distance of 6 Earth radii can appreciably alter the spin state of 99942 Apophis. More distant encounters could still excite the spin state enough to induce seismic activity, leading to a periodic resurfacing of the asteroid that may have implications for interpretation of Bennu samples returned by OSIRIS-REx.

We have seen that the current levels of uncertainty in Bennu's orbit are low enough that unprecedented levels of accuracy are required in the dynamical model that governs the trajectory. For example, the relativistic perturbation of planetary gravity fields, in particular that of Earth, must be incorporated to obtain reliable results. The future addition of OSIRIS-REx radio science data will again decrease the orbital uncertainties by 1-2 orders of magnitude, which will likely require even finer scale refinements to our dynamical model than used here. However, difficulties in understanding the proper statistical treatment of asteroid optical astrometry, and in particular the identification of statistical outliers, will likely remain a dominant source of uncertainty that is not properly captured by a posteriori covariance analysis.

Thus our findings for the post-OSIRIS-REx orbital uncertainties of Bennu may be illusory. The finding that $\zeta_{2135}$ uncertainties may be reduced as low as $1000 \mathrm{~km}$ with the OSIRIS-REx radio science data assumes that our Yarkovsky model, including the asteroid spin state, holds through 2135. Thus a host of model refinements may be necessary to properly characterize the trajectory out to 2135. Notwithstanding the next radar observation opportunity in January 2037, we may reach an uncertainty limit that prevents us from improving predictions any further until models can improve or the prediction interval is significantly reduced. As an example, the post-2135 predictability will markedly improve after the 0.005 au Earth close approach in 2060, and it is reasonable to expect that at least some potential impacts will persist until that time.

\section{Acknowledgments}

We are grateful to Giovanni F. Gronchi (Univ. Pisa) for his assistance in calculating Bennu's orbital precession period and its variability.

This research was conducted in part at the Jet Propulsion Laboratory, California Institute of Technology, under a contract with the National Aeronautics and Space Administration.

D.F. was supported in part by an appointment to the NASA Postdoctoral Program at the Jet 
Propulsion Laboratory, California Institute of Technology, administered by Oak Ridge Associated Universities through a contract with NASA. Prior to Sept. 2012 his support was through SpaceDys s.r.l. under a contract with the European Space Agency.

The work of D.V. was partially supported by the Czech Grant Agency (grant P209-13-01308S).

The work of B.R. is supported by the UK Science and Technology Facilities Council (STFC).

The Arecibo Observatory is operated by SRI International under a cooperative agreement with the National Science Foundation (AST-1100968), and in alliance with Ana G. Mendez-Universidad Metropolitana, and the Universities Space Research Association. At the time of the observations used in this paper, the Arecibo Observatory was operated by Cornell University under a cooperative agreement with NSF and with support from NASA.

The Arecibo Planetary Radar Program is supported by the National Aeronautics and Space Administration under Grant No. NNX12AF24G issued through the Near Earth Object Observations program.

This research was supported in part by NASA under the Science Mission Directorate Research and Analysis Program.

\section{REFERENCES}

Baer, J. and Chesley, S. R. (2008). Astrometric masses of 21 asteroids, and an integrated asteroid ephemeris. Celestial Mechanics and Dynamical Astronomy, 100:27-42.

Baer, J., Chesley, S. R., and Matson, R. D. (2011). Astrometric Masses of 26 Asteroids and Observations on Asteroid Porosity. AJ, 141:143.

Bottke, Jr., W. F., Vokrouhlický, D., Rubincam, D. P., and Nesvorný, D. (2006). The Yarkovsky and Yorp Effects: Implications for Asteroid Dynamics. Annual Review of Earth and Planetary Sciences, 34:157-191.

Campins, H., Morbidelli, A., Tsiganis, K., de León, J., Licandro, J., and Lauretta, D. (2010). The Origin of Asteroid 101955 (1999 RQ36). ApJ, 721:L53-L57.

Čapek, D. and Vokrouhlický, D. (2005). Accurate model for the Yarkovsky effect. In Knežević, Z. and Milani, A., editors, IAU Colloq. 19\%: Dynamics of Populations of Planetary Systems, pages $171-178$.

Carpino, M., Milani, A., and Chesley, S. R. (2003). Error statistics of asteroid optical astrometric observations. Icarus, 166:248-270.

Carry, B. (2012). Density of asteroids. Planet. Space Sci., 73:98-118. 
Chesley, S. R. (2006). Potential impact detection for Near-Earth asteroids: the case of 99942 Apophis (2004 MN 4 ). In Lazzaro, D., Ferraz-Mello, S., and Fernández, J. A., editors, Asteroids, Comets, Meteors, volume 229 of IAU Symposium, pages 215-228.

Chesley, S. R., Baer, J., and Monet, D. G. (2010). Treatment of star catalog biases in asteroid astrometric observations. Icarus, 210:158-181.

Chesley, S. R., Chodas, P. W., Milani, A., Valsecchi, G. B., and Yeomans, D. K. (2002). Quantifying the risk posed by potential Earth impacts. Icarus, 159:423-432.

Chesley, S. R., Nolan, M. C., Farnocchia, D., Milani, A., Emery, J., Vokrouhlicky, D., Lauretta, D. S., Taylor, P. A., Benner, L. A. M., Giorgini, J. D., Brozovic, M., Busch, M. W., Margot, J.-L., Howell, E. S., Naidu, S. P., Valsecchi, G. B., and Bernardi, F. (2012). The Trajectory Dynamics of Near-Earth Asteroid 101955 (1999 RQ36). LPI Contributions, 1667:6470.

Chesley, S. R., Ostro, S. J., Vokrouhlický, D., Čapek, D., Giorgini, J. D., Nolan, M. C., Margot, J.-L., Hine, A. A., Benner, L. A. M., and Chamberlin, A. B. (2003). Direct Detection of the Yarkovsky Effect by Radar Ranging to Asteroid 6489 Golevka. Science, 302:1739-1742.

Chesley, S. R., Vokrouhlický, D., Ostro, S. J., Benner, L. A. M., Margot, J.-L., Matson, R. L., Nolan, M. C., and Shepard, M. K. (2008). Direct Estimation of Yarkovsky Accelerations on Near-Earth Asteroids. LPI Contributions, 1405:8330.

Chodas, P. W. (1999). Orbit uncertainties, keyholes, and collision probabilities. BAAS, 31:2804.

Chodas, P. W. and Yeomans, D. K. (1999). Predicting close approaches and estimating impact probabilities for near-Earth objects. Paper AAS 99-462, AAS/AIAA Astrodynamics Specialists Conference, Girdwood, Alaska.

Clark, B. E., Binzel, R. P., Howell, E. S., Cloutis, E. A., Ockert-Bell, M., Christensen, P., Barucci, M. A., DeMeo, F., Lauretta, D. S., Connolly, H., Soderberg, A., Hergenrother, C., Lim, L., Emery, J., and Mueller, M. (2011). Asteroid (101955) 1999 RQ36: Spectroscopy from 0.4 to $2.4 \mu \mathrm{m}$ and meteorite analogs. Icarus, 216:462-475.

Consolmagno, G., Britt, D., and Macke, R. (2008). The significance of meteorite density and porosity. Chemie der Erde / Geochemistry, 68:1-29.

Damour, T., Soffel, M., and Xu, C. (1994). General-relativistic celestial mechanics. IV. Theory of satellite motion. Phys. Rev. D, 49:618-635.

Delbò, M. and Michel, P. (2011). Temperature History and Dynamical Evolution of (101955) 1999 RQ 36: A Potential Target for Sample Return from a Primitive Asteroid. ApJ, 728:L42.

Durda, D. D., Bottke, W. F., Enke, B. L., Merline, W. J., Asphaug, E., Richardson, D. C., and Leinhardt, Z. M. (2004). The formation of asteroid satellites in large impacts: results from numerical simulations. Icarus, 170:243-257. 
Emery, J. P., Fernández, Y. R., Kelley, M. S., Hergenrother, C., Ziffer, J., Lauretta, D. S., Drake, M. J., and Campins, H. (2010). Thermophysical Characterization of Potential Spacecraft Target (101955) 1999 RQ36. In Lunar and Planetary Institute Science Conference Abstracts, volume 41 of Lunar and Planetary Institute Science Conference Abstracts, page 2282.

Emery, J. P., Fernandez, Y. R., Kelley, M. S. P., Warden, K. T., Hergenrother, C. W., Lauretta, D. S., Drake, M. J., Campins, H., and Ziffer, J. (2014). Thermal Infrared Observations and Thermophysical Characterization of OSIRIS-REx Target Asteroid (101955) Bennu. Icarus. In press.

Emery, J. P., Kelley, M. S., Fernandez, Y. R., Hergenrother, C. W., Crane, K. T., Ziffer, J., Campins, H., Lauretta, D. S., and Drake, M. J. (2012). Thermal and Physical Characterization of the OSIRIS-REx Target Asteroid (101955) 1999 RQ36. In AAS/Division for Planetary Sciences Meeting Abstracts, volume 44 of AAS/Division for Planetary Sciences Meeting Abstracts, page 102.05.

Farnocchia, D. and Chesley, S. R. (2014). Assessment of the 2880 impact threat from Asteroid (29075) 1950 DA. Icarus, 229:321-327.

Farnocchia, D., Chesley, S. R., Chodas, P. W., Micheli, M., Tholen, D. J., Milani, A., Elliott, G. T., and Bernardi, F. (2013a). Yarkovsky-driven impact risk analysis for asteroid (99942) Apophis. Icarus, 224:192-200.

Farnocchia, D., Chesley, S. R., Vokrouhlický, D., Milani, A., Spoto, F., and Bottke, W. F. (2013b). Near Earth Asteroids with measurable Yarkovsky effect. Icarus, 224:1-13.

Folkner, W. M. (2011). Planetary ephemeris DE424 for Mars Science Laboratory early cruise navigation. Technical Report IOM 343R-11-003, Jet Propulsion Laboratory.

Giorgini, J. D., Benner, L. A. M., Ostro, S. J., Nolan, M. C., and Busch, M. W. (2008). Predicting the Earth encounters of (99942) Apophis. Icarus, 193:1-19.

Giorgini, J. D., Ostro, S. J., Benner, L. A. M., Chodas, P. W., Chesley, S. R., Hudson, R. S., Nolan, M. C., Klemola, A. R., Standish, E. M., Jurgens, R. F., Rose, R., Chamberlin, A. B., Yeomans, D. K., and Margot, J.-L. (2002). Asteroid 1950 DA's Encounter with Earth in 2880: Physical Limits of Collision Probability Prediction. Science, 296:132-136.

Gronchi, G. F. (2005). An Algebraic Method to Compute the Critical Points of the Distance Function Between Two Keplerian Orbits. Celestial Mechanics and Dynamical Astronomy, 93:295-329.

Jewitt, D. and Li, J. (2010). Activity in Geminid Parent (3200) Phaethon. AJ, 140:1519-1527.

Konopliv, A. S., Asmar, S. W., Folkner, W. M., Karatekin, Ö., Nunes, D. C., Smrekar, S. E., Yoder, C. F., and Zuber, M. T. (2011). Mars high resolution gravity fields from MRO, Mars seasonal gravity, and other dynamical parameters. Icarus, 211:401-428. 
Marchis, F., Descamps, P., Baek, M., Harris, A. W., Kaasalainen, M., Berthier, J., Hestroffer, D., and Vachier, F. (2008a). Main belt binary asteroidal systems with circular mutual orbits. Icarus, 196:97-118.

Marchis, F., Descamps, P., Berthier, J., Hestroffer, D., Vachier, F., Baek, M., Harris, A. W., and Nesvorný, D. (2008b). Main belt binary asteroidal systems with eccentric mutual orbits. Icarus, 195:295-316.

Milani, A., Chesley, S. R., Sansaturio, M. E., Bernardi, F., Valsecchi, G. B., and Arratia, O. (2009). Long term impact risk for (101955) 1999 RQ. Icarus, 203:460-471.

Milani, A., Chesley, S. R., Sansaturio, M. E., Tommei, G., and Valsecchi, G. B. (2005). Nonlinear impact monitoring: line of variation searches for impactors. Icarus, 173:362-384.

Moyer, T. D. (2003). Formulation for Observed and Computed Values of Deep Space Network Data Types for Navigation. Wiley-Interscience, Hoboken, NJ.

Müller, T. G., O’Rourke, L., Barucci, A. M., Pál, A., Kiss, C., Zeidler, P., Altieri, B., GonzálezGarcía, B. M., and Küppers, M. (2012). Physical properties of OSIRIS-REx target asteroid (101955) 1999 RQ36. Derived from Herschel, VLT/ VISIR, and Spitzer observations. A\&A, 548:A36.

Müller, T. G., Sekiguchi, T., Kaasalainen, M., Abe, M., and Hasegawa, S. (2005). Thermal infrared observations of the Hayabusa spacecraft target asteroid 25143 Itokawa. A\&A, 443:347-355.

Nesvorný, D., Bottke, W. F., Vokrouhlický, D., Chapman, C. R., and Rafkin, S. (2010). Do planetary encounters reset surfaces of near Earth asteroids? Icarus, 209:510-519.

Nolan, M. C., Magri, C., Howell, E. S., Benner, L. A. M., Giorgini, J. D., Hergenrother, C. W., Hudson, R. S., Lauretta, D. S., Margot, J.-L., Ostro, S. J., and Scheeres, D. J. (2013). Shape Model and Surface Properties of the OSIRIS-REx Target Asteroid (101955) Bennu from Radar and Lightcurve Observations. Icarus, In press.

Nugent, C. R., Margot, J. L., Chesley, S. R., and Vokrouhlický, D. (2012). Detection of Semimajor Axis Drifts in 54 Near-Earth Asteroids: New Measurements of the Yarkovsky Effect. AJ, 144:60.

Richardson, D. C., Leinhardt, Z. M., Melosh, H. J., Bottke, Jr., W. F., and Asphaug, E. (2002). Gravitational Aggregates: Evidence and Evolution. Asteroids III, pages 501-515.

Rozitis, B., Duddy, S. R., Green, S. F., and Lowry, S. C. (2013). A thermophysical analysis of the (1862) Apollo Yarkovsky and YORP effects. A\&A, 555:A20.

Rozitis, B. and Green, S. F. (2012). The influence of rough surface thermal-infrared beaming on the Yarkovsky and YORP effects. MNRAS, 423:367-388. 
Russell, C. T., Raymond, C. A., Coradini, A., McSween, H. Y., Zuber, M. T., Nathues, A., De Sanctis, M. C., Jaumann, R., Konopliv, A. S., Preusker, F., Asmar, S. W., Park, R. S., Gaskell, R., Keller, H. U., Mottola, S., Roatsch, T., Scully, J. E. C., Smith, D. E., Tricarico, P., Toplis, M. J., Christensen, U. R., Feldman, W. C., Lawrence, D. J., McCoy, T. J., Prettyman, T. H., Reedy, R. C., Sykes, M. E., and Titus, T. N. (2012). Dawn at Vesta: Testing the Protoplanetary Paradigm. Science, 336:684-686.

Scheeres, D. J., Benner, L. A. M., Ostro, S. J., Rossi, A., Marzari, F., and Washabaugh, P. (2005). Abrupt alteration of the spin state of asteroid 99942 Apophis (2004 MN4) during its 2029 Earth flyby. Icarus, 175:281-283.

Shepard, M. K., Margot, J.-L., Magri, C., Nolan, M. C., Schlieder, J., Estes, B., Bus, S. J., Volquardsen, E. L., Rivkin, A. S., Benner, L. A. M., Giorgini, J. D., Ostro, S. J., and Busch, M. W. (2006). Radar and infrared observations of binary near-Earth Asteroid 2002 CE26. Icarus, 184:198-210.

Soffel, M., Klioner, S. A., Petit, G., Wolf, P., Kopeikin, S. M., Bretagnon, P., Brumberg, V. A., Capitaine, N., Damour, T., Fukushima, T., Guinot, B., Huang, T.-Y., Lindegren, L., Ma, C., Nordtvedt, K., Ries, J. C., Seidelmann, P. K., Vokrouhlický, D., Will, C. M., and Xu, C. (2003). The IAU 2000 Resolutions for Astrometry, Celestial Mechanics, and Metrology in the Relativistic Framework: Explanatory Supplement. AJ, 126:2687-2706.

Standish, E. M. (2000). Recommendation of DE405 for 2001 Mars Surveyor and for Cassini. Technical Report IOM 312.F-00-107b, Jet Propulsion Laboratory.

Valsecchi, G. B., Milani, A., Gronchi, G. F., and Chesley, S. R. (2003). Resonant returns to close approaches: Analytical theory. A\&\&A, 408:1179-1196.

Vokrouhlický, D., Milani, A., and Chesley, S. R. (2000). Yarkovsky Effect on Small Near-Earth Asteroids: Mathematical Formulation and Examples. Icarus, 148:118-138.

Vokrouhlický, D. (1998). Diurnal Yarkovsky effect as a source of mobility of meter-sized asteroidal fragments. II. Non-sphericity effects. A\&A, 338:353-363.

Vokrouhlický, D., Chesley, S. R., and Matson, R. D. (2008). Orbital Identification for Asteroid 152563 (1992 Bf) Through the Yarkovsky Effect. AJ, 135:2336-2340.

Vokrouhlický, D. and Milani, A. (2000). Direct solar radiation pressure on the orbits of small near-Earth asteroids: observable effects? A\&A, 362:746-755.

Walsh, K. J., Delbó, M., Bottke, W. F., Vokrouhlický, D., and Lauretta, D. S. (2013). Introducing the Eulalia and new Polana asteroid families: Re-assessing primitive asteroid families in the inner Main Belt. Icarus, 225:283-297.

Walsh, K. J. and Richardson, D. C. (2008). A steady-state model of NEA binaries formed by tidal disruption of gravitational aggregates. Icarus, 193:553-566. 
Whipple, F. L. (1983). 1983 TB and the Geminid Meteors. IAU Circ., 3881:1.

Will, C. M. (1993). Theory and Experiment in Gravitational Physics. Cambridge Univ. Press, Cambridge.

Yeomans, D. K., Barriot, J.-P., Dunham, D. W., Farquhar, R. W., Giorgini, J. D., Helfrich, C. E., Konopliv, A. S., McAdams, J. V., Miller, J. K., Owen, W., Scheeres, D. J., Synnott, S. P., and Williams, B. G. (1997). Estimating the mass of asteroid 253 Mathilde from tracking data during the NEAR flyby. Science, 278:2106-2109.

Yeomans, D. K., Chodas, P. W., Keesey, M. S., Ostro, S. J., Chandler, J. F., and Shapiro, I. I. (1992). Asteroid and comet orbits using radar data. AJ, 103:303-317.

\section{A. Finding the best power law to model the Yarkovsky effect}

We want to find the value of $d$ such that the transverse acceleration $A_{T}\left(r / r_{0}\right)^{-d}, r_{0}=1$ au, provides the best match to the Yarkovsky acceleration acting on Bennu. We can neglect the seasonal component of the Yarkovsky effect. In fact, the diurnal component is usually the dominant one (Vokrouhlický et al. 2000). Moreover, the obliquity of Bennu is $175^{\circ}$ and the seasonal component vanishes when the spin axis is normal to the orbital plane (Bottke et al. 2006). For the diurnal

component of the Yarkovsky effect we have that the transverse acceleration is (Vokrouhlický 1998)

$$
a_{t}=\frac{4(1-A)}{9} \phi(r) f(\Theta) \cos (\gamma), f(\Theta)=\frac{0.5 \Theta}{1+\Theta+0.5 \Theta^{2}}
$$

where $A$ is the Bond albedo, $\phi$ the standard radiation force factor at heliocentric distance $r, \Theta$ the thermal parameter, and $\gamma$ the obliquity.

The dependence on $r$ is contained in $\phi(r) \propto r^{-2}$ and $f(\Theta)$. As a matter of fact, $\Theta$ depends on the subsolar temperature $T_{\star}$, and $T_{\star}$ depends on $r$ :

$$
\Theta=\frac{\Gamma}{\epsilon \sigma T_{\star}^{3}} \sqrt{\frac{2 \pi}{P}}, T_{\star}=\sqrt[4]{\frac{(1-A) G_{S}}{\sigma \epsilon}\left(\frac{1 \mathrm{au}}{r}\right)^{2}}
$$

where $\Gamma$ is the thermal inertia, $\epsilon$ the emissivity, $\sigma$ the Stefan-Boltzmann constant, $G_{S}=1365 \mathrm{~W} / \mathrm{m}^{2}$ is the solar constant, and $P$ the rotation period. Thus, $\Theta \propto r^{3 / 2}$.

We want to approximate $f(\Theta)$ with a power law $\left(r / r_{0}\right)^{\psi}$ :

$$
f(\Theta) \simeq c\left(r / r_{0}\right)^{\psi} \Longrightarrow \log f(\Theta) \simeq \log c+\psi \log \left(r / r_{0}\right) .
$$

This preprint was prepared with the AAS LATEX macros v5.2. 
By differentiating with respect to $r$ we find:

$$
\psi=\left.r \frac{\partial \log f(\Theta)}{\partial r}\right|_{r=r_{0}}
$$

From the chain rule we obtain

$$
\frac{\partial \log f(\Theta)}{\partial r}=\frac{1}{f(\Theta)} \frac{\partial f(\Theta)}{\partial \Theta} \frac{\partial \Theta}{\partial r}
$$

Evaluating this equation for $r=r_{0}$ and using the physical quantities as in Table 4 yield $\psi \simeq-0.24$. Therefore, $d \simeq 2.24$, which is in good agreement with the value 2.25 found numerically in Sec. 3.1 .

[Figure 16 about here.]

(C)2014. All rights reserved. 


\section{List of Figures}

1 Depiction of Bennu postfit residuals for JPL solution 87. Deleted observations are depicted with circles around the points. In addition to the observations shown here, there are 14 deleted observations outside the plot boundaries. . . . . . . . . . . 33

2 Arecibo delay-Doppler detections of Bennu from 2011-Sep-27,28,29. Doppler frequency increases to the right and delay increases upwards. Image resolution is

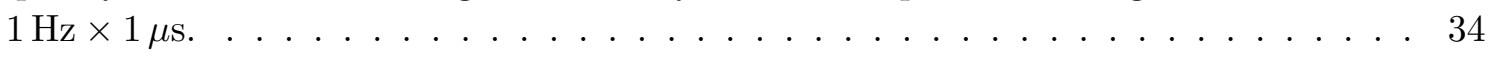

3 Time history of the osculating Bennu orbital elements, $a, e$ and $i$, through 2136 . The effects of Earth encounters are evident and can be cross-referenced with Table 8. . . 35

4 Time history of the Bennu semimajor axis drift $d a / d t$ through 2136. The variations arise from orbital changes induced by Earth encounters as depicted in Fig. 3. . . . . 36

5 Yarkovsky accelerations as a function of heliocentric distance, according to the linear and nonlinear Yarkovsky models. . . . . . . . . . . . . . . 37

6 The history of $d a / d t$ stemming from the transverse and radial components of the Yarkovsky acceleration. The linear Yarkovsky model is depicted. . . . . . . . . . 38

7 Comparison of the Yarkovsky effect with the relativistic perturbations on Bennu. The magnitude of the respective accelerations is plotted. . . . . . . . . . . 39

8 Comparison of the transverse perturbations from the Yarkovsky effect and the relativistic component due to the Earth. Earth relativity can be significantly greater in magnitude than the Yarkovsky effect during Earth encounters, and thus provides a statistically significant change in semimajor axis that must be accounted for in the dynamical model. . . . . . . . . . . . . . . . . . . . 40 40

9 The bulk density estimate for Bennu depends nonlinearly on the estimated thermal inertia $\Gamma$. Neglecting surface roughness, we obtain a bulk density estimate of 1180 $\mathrm{kg} / \mathrm{m}^{3}$. However, taking into account the assumed Yarkovsky enhancement from roughness, as well as uncertainties in obliquity, diameter and thermal inertia, we obtain $1260 \pm 70 \mathrm{~kg} / \mathrm{m}^{3}$ as depicted here. . . . . . . . . . . . . 41

10 Yarkovsky effect enhancement due to $100 \%$ surface roughness as a function of thermal

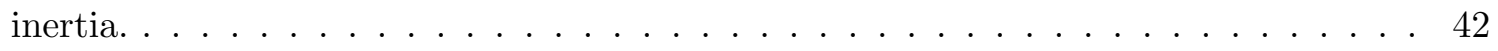

11 Time history of the MOID. The Earth impact cross-section (2.1 Earth radii) is marked by the dashed line. . . . . . . . . . . . . . . . . 43

12 Uncertainty region on the $b$-plane of the 2135 encounter. The Earth is plotted to scale at the origin, and the impact cross-section is indicated by the dots. . . . . . . 44 
13 A map of the Bennu impact keyholes on the 2135 b-plane. The probability density is given by the curve with the left ordinate, and the keyholes are indicated by the vertical lines at their respective $\zeta_{2135}$ positions with their widths given on the right ordinate. For clarity only keyholes wider than $1 \mathrm{~km}$ are labeled with the year of impact and only keyholes $>100 \mathrm{~m}$ in width are depicted. Potential impacts with impact probability greater than $10^{-5}$ are marked with a circle at the top of the vertical bar. . . . . . . . . . . . . . . . . . . 45

14 Cumulative impact probability (upper) and cumulative number of keyholes found (lower), each as a function of diminishing keyhole width. The cumulative impact probability over all potential impacts is $3.7 \times 10^{-4} \ldots \ldots \ldots$. . . . . . . . 46

15 Probability (with corresponding $1 \sigma$ error bars) of having at least one close approach (CA) within a given distance for different time intervals. The dashed curve is for a node crossing cycle, while solid line are for $1 \mathrm{yr}, 1000 \mathrm{yr}$, and 1,000,000 yr. The Earth radius $\left(R_{\oplus}\right)$ and lunar distance (LD) are marked by vertical lines. . . . . . . . 47

16 Dependency of transverse acceleration exponent $d$ on thermal inertia $\Gamma$ for Bennu. . 48 


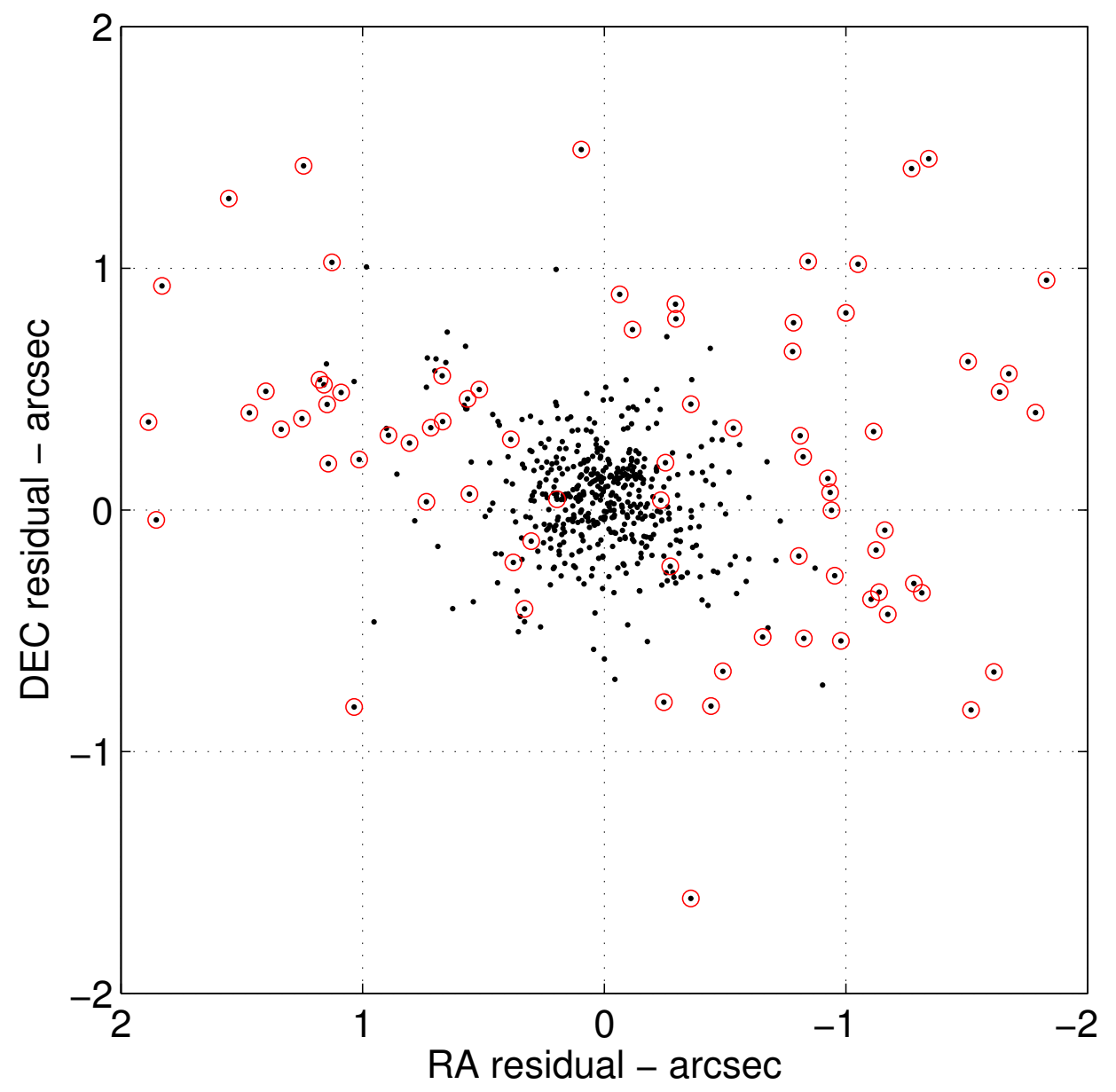

Fig. 1.- Depiction of Bennu postfit residuals for JPL solution 87. Deleted observations are depicted with circles around the points. In addition to the observations shown here, there are 14 deleted observations outside the plot boundaries. 


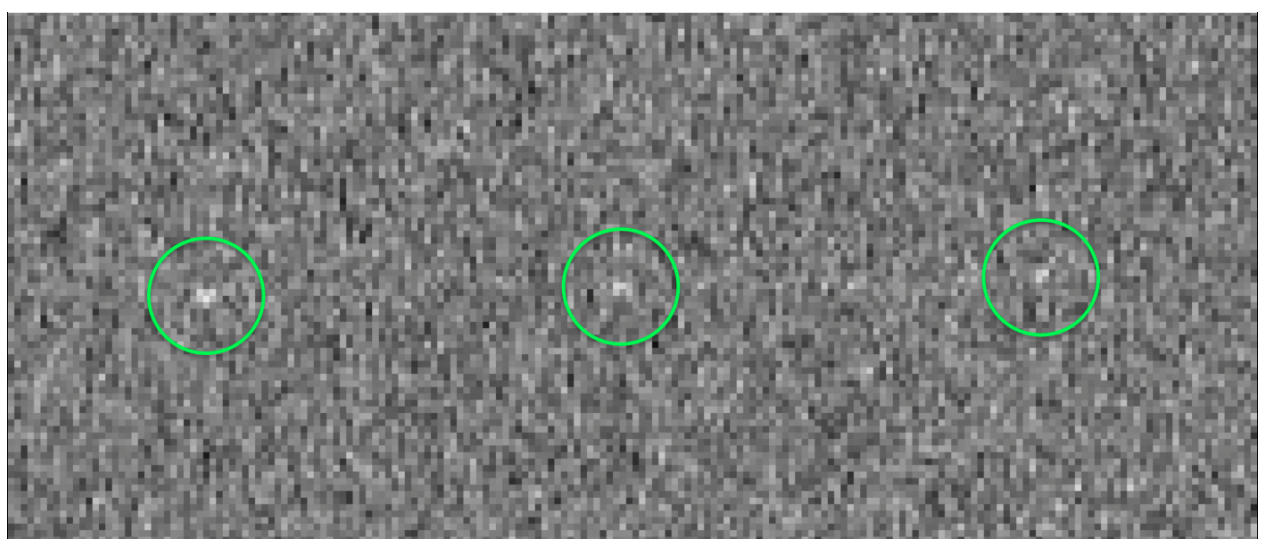

Fig. 2.- Arecibo delay-Doppler detections of Bennu from 2011-Sep-27,28,29. Doppler frequency increases to the right and delay increases upwards. Image resolution is $1 \mathrm{~Hz} \times 1 \mu \mathrm{s}$. 

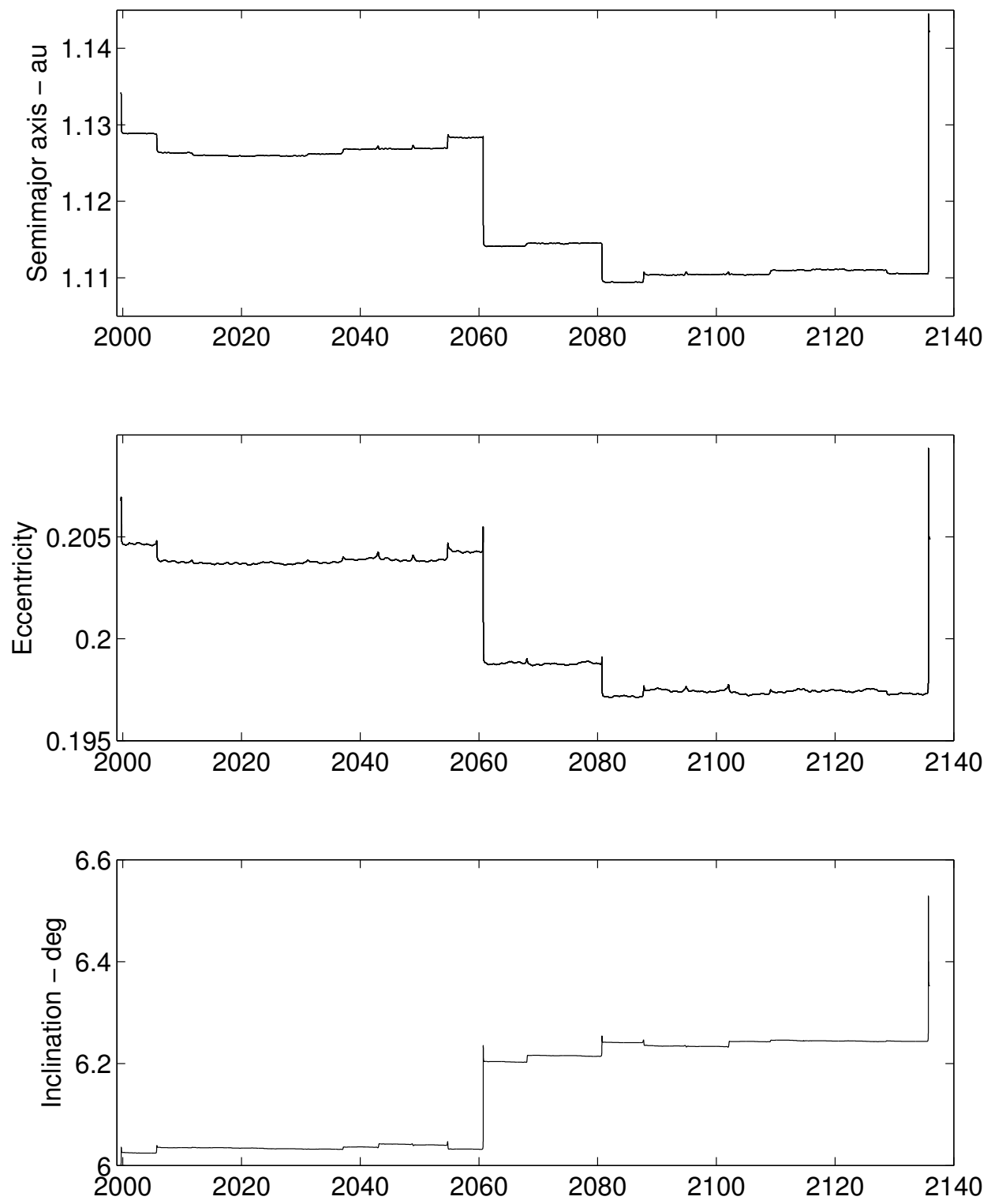

Fig. 3.- Time history of the osculating Bennu orbital elements, $a, e$ and $i$, through 2136. The effects of Earth encounters are evident and can be cross-referenced with Table 8 . 


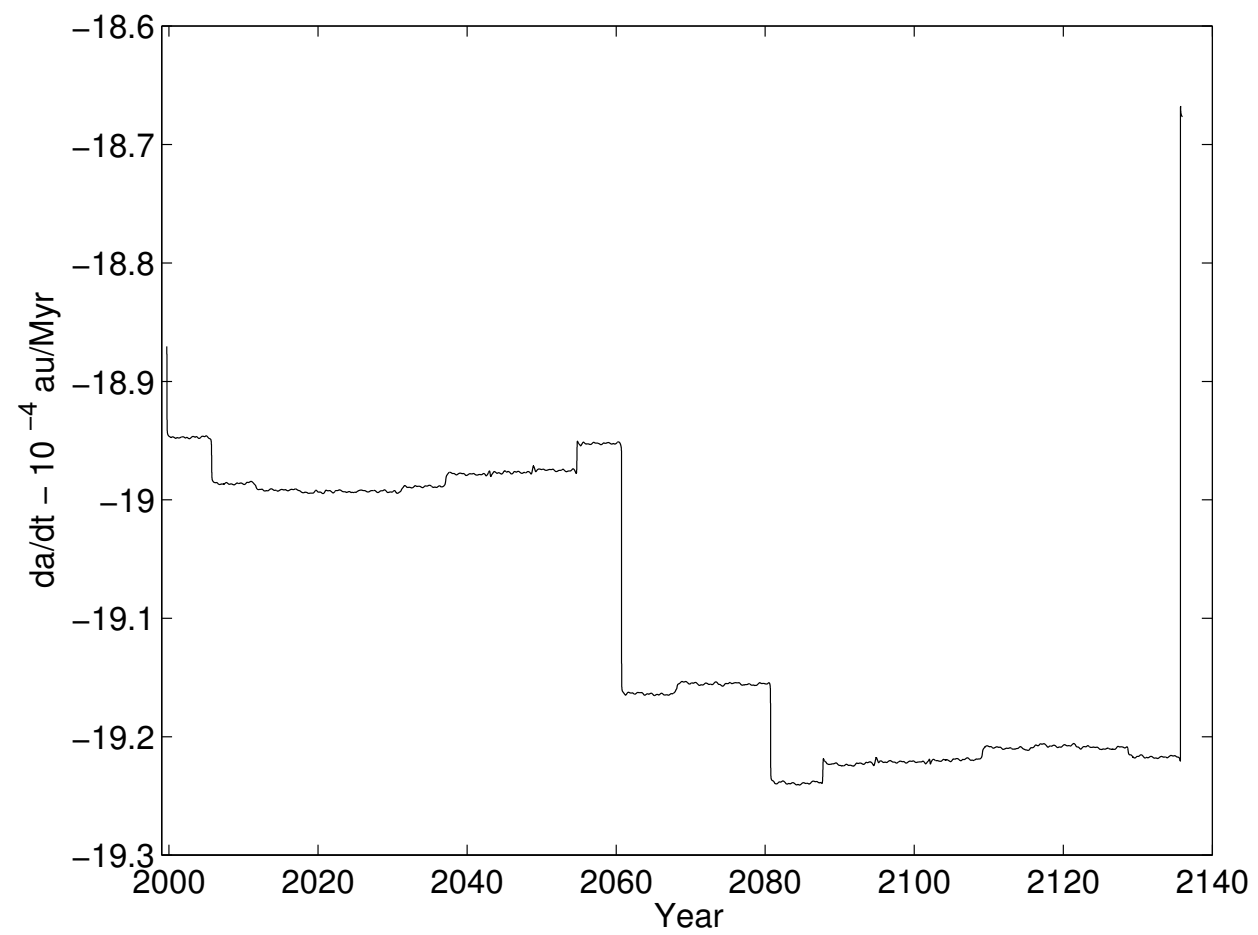

Fig. 4.- Time history of the Bennu semimajor axis drift $d a / d t$ through 2136. The variations arise from orbital changes induced by Earth encounters as depicted in Fig. 3 . 


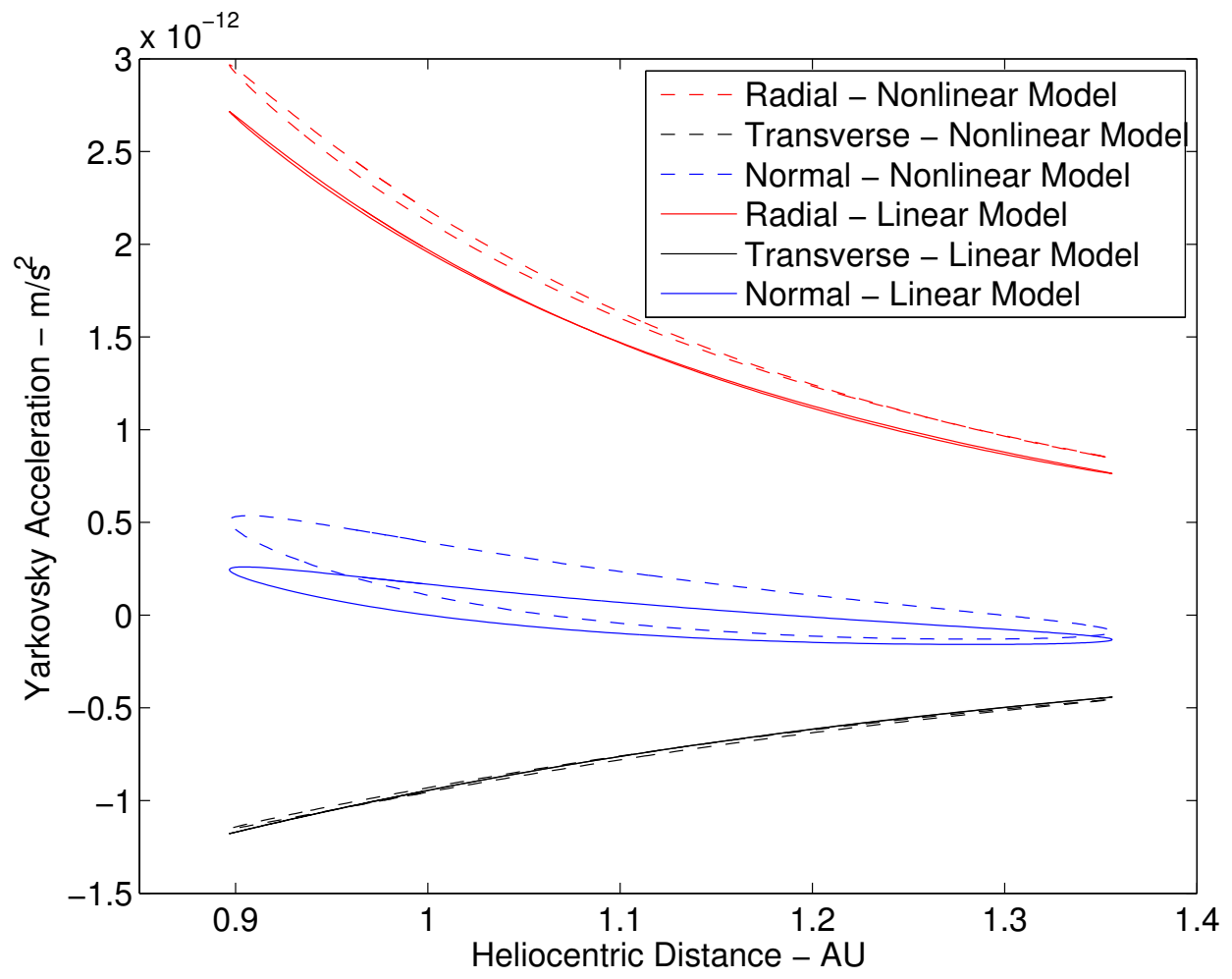

Fig. 5.- Yarkovsky accelerations as a function of heliocentric distance, according to the linear and nonlinear Yarkovsky models. 

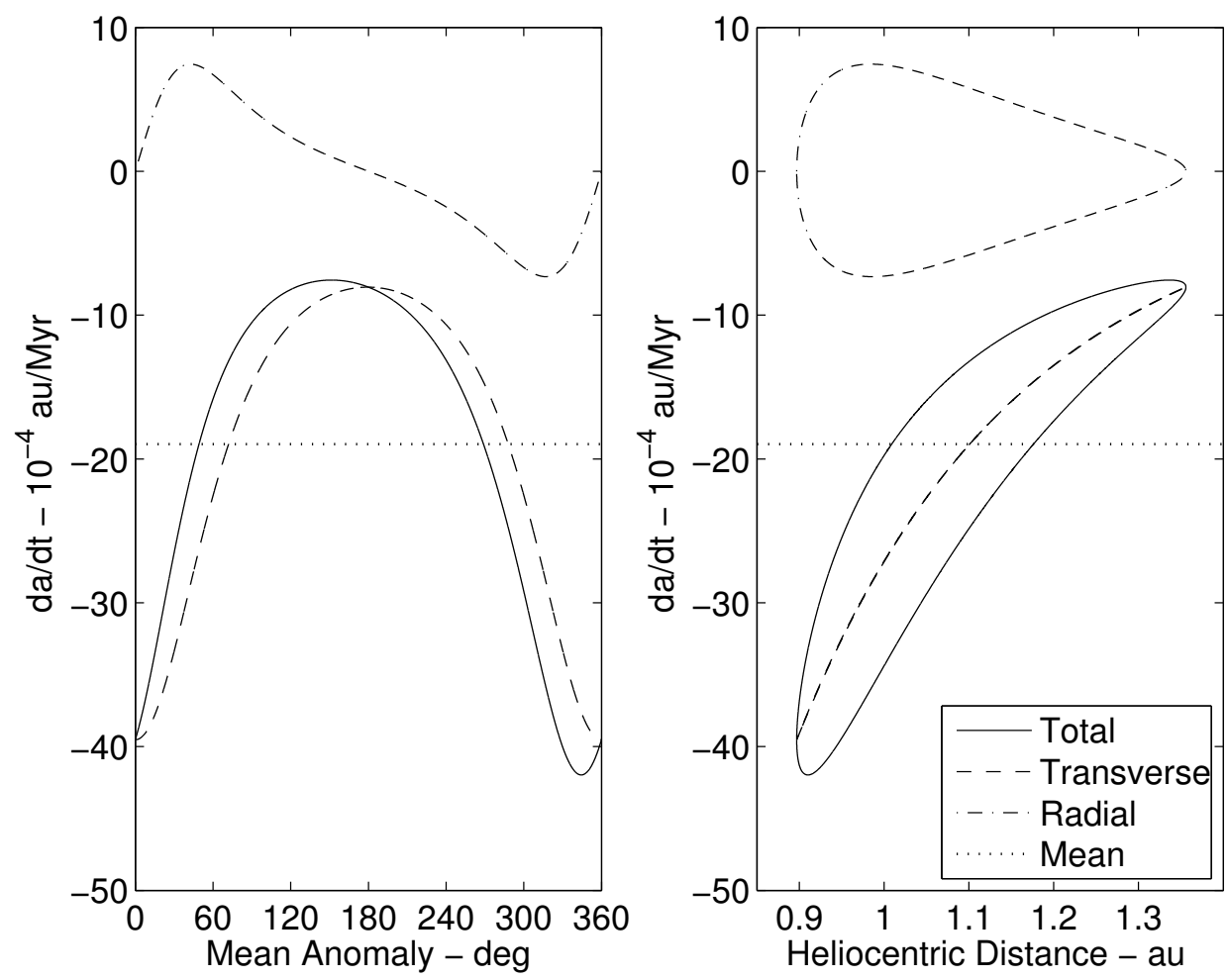

Fig. 6. - The history of $d a / d t$ stemming from the transverse and radial components of the Yarkovsky acceleration. The linear Yarkovsky model is depicted. 


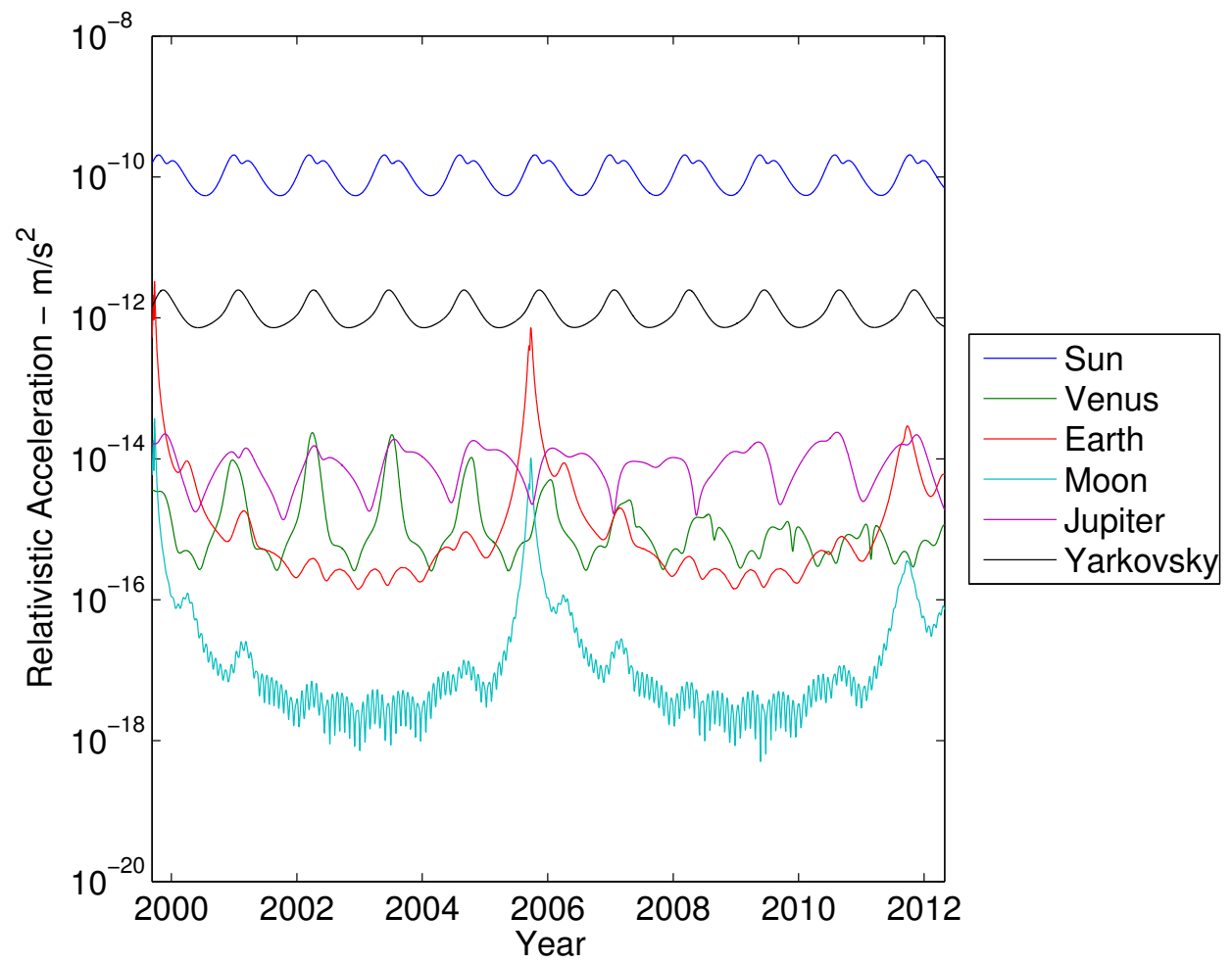

Fig. 7.- Comparison of the Yarkovsky effect with the relativistic perturbations on Bennu. The magnitude of the respective accelerations is plotted. 


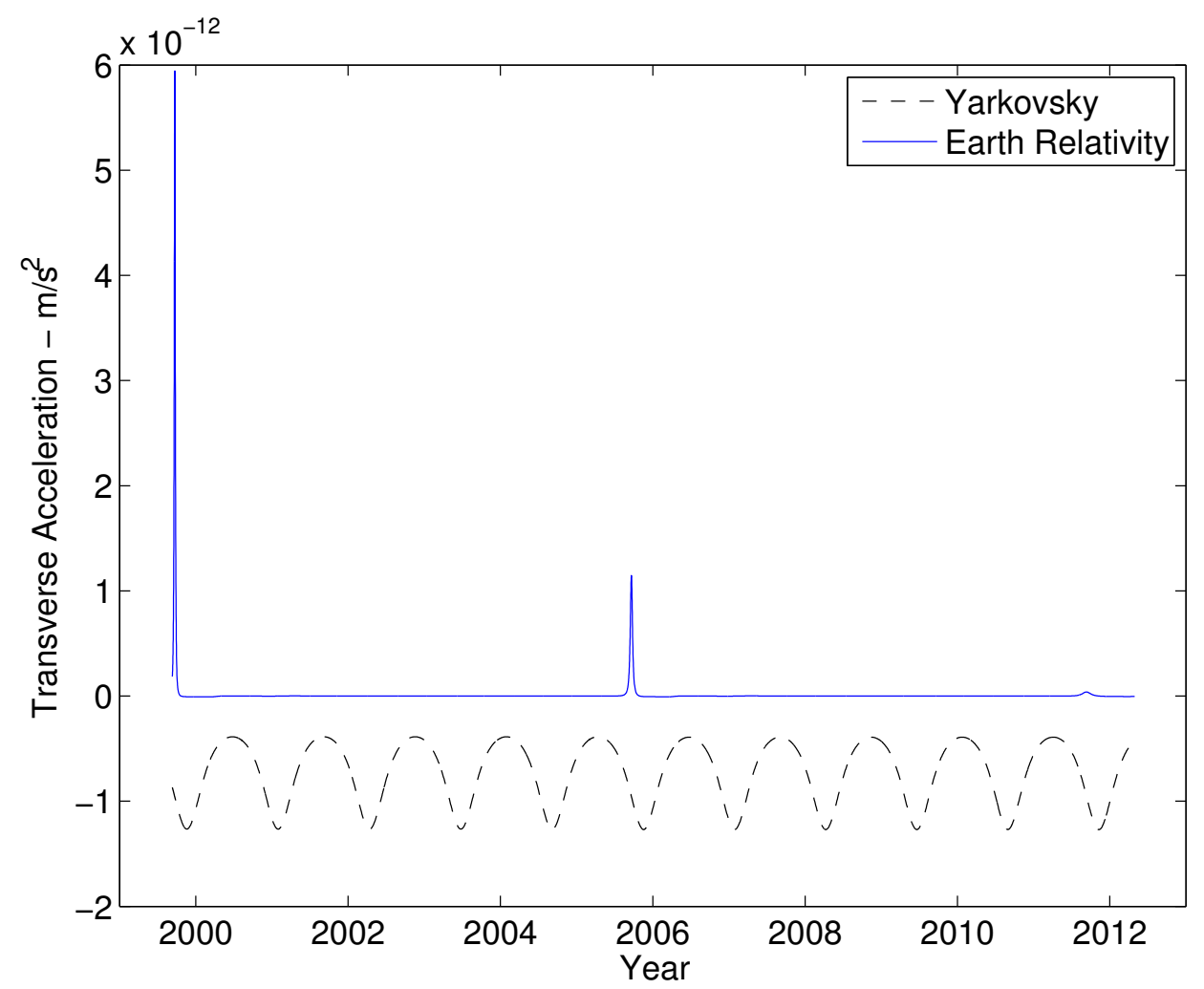

Fig. 8.- Comparison of the transverse perturbations from the Yarkovsky effect and the relativistic component due to the Earth. Earth relativity can be significantly greater in magnitude than the Yarkovsky effect during Earth encounters, and thus provides a statistically significant change in semimajor axis that must be accounted for in the dynamical model. 


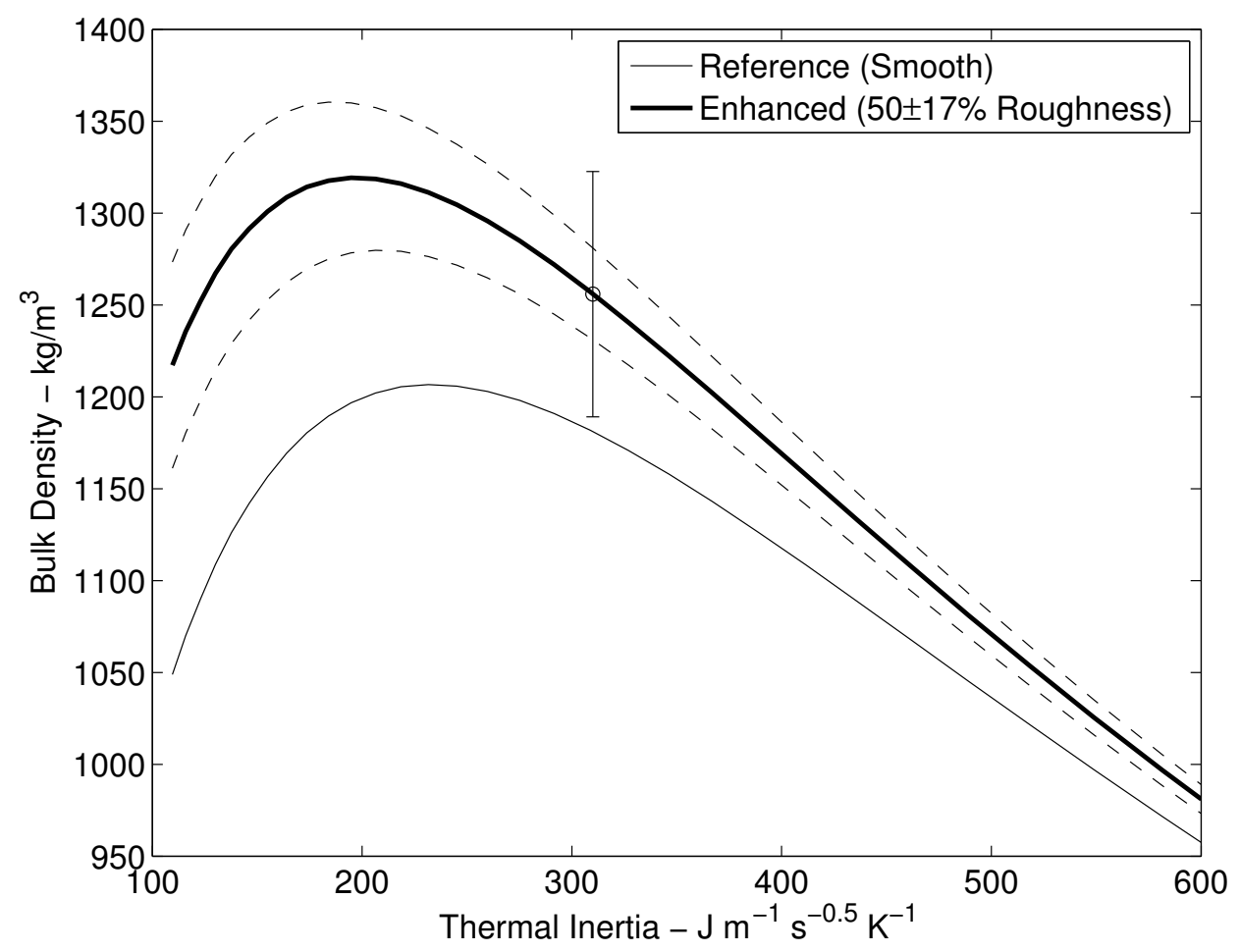

Fig. 9.- The bulk density estimate for Bennu depends nonlinearly on the estimated thermal inertia $\Gamma$. Neglecting surface roughness, we obtain a bulk density estimate of $1180 \mathrm{~kg} / \mathrm{m}^{3}$. However, taking into account the assumed Yarkovsky enhancement from roughness, as well as uncertainties in obliquity, diameter and thermal inertia, we obtain $1260 \pm 70 \mathrm{~kg} / \mathrm{m}^{3}$ as depicted here. 


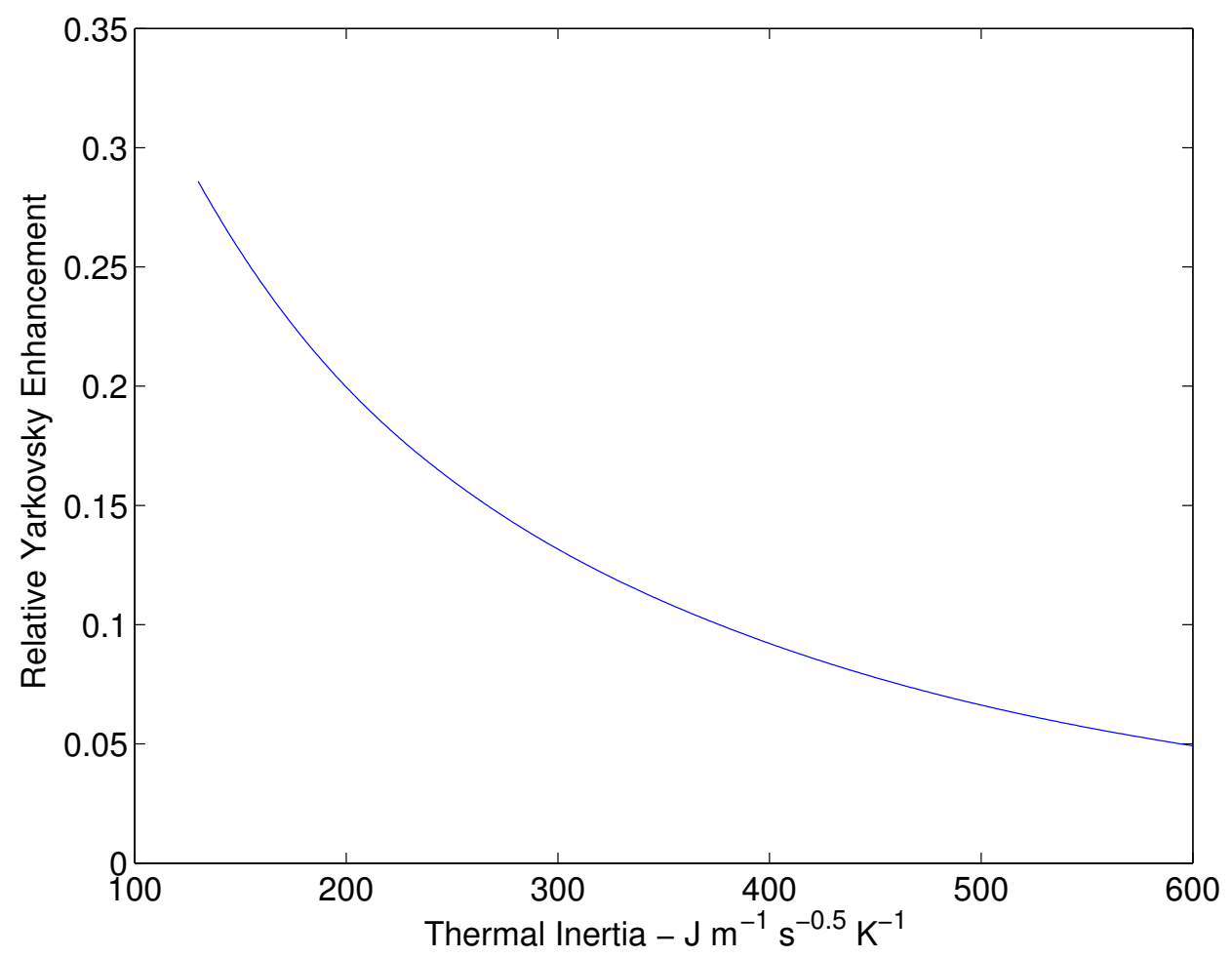

Fig. 10.- Yarkovsky effect enhancement due to $100 \%$ surface roughness as a function of thermal inertia. 


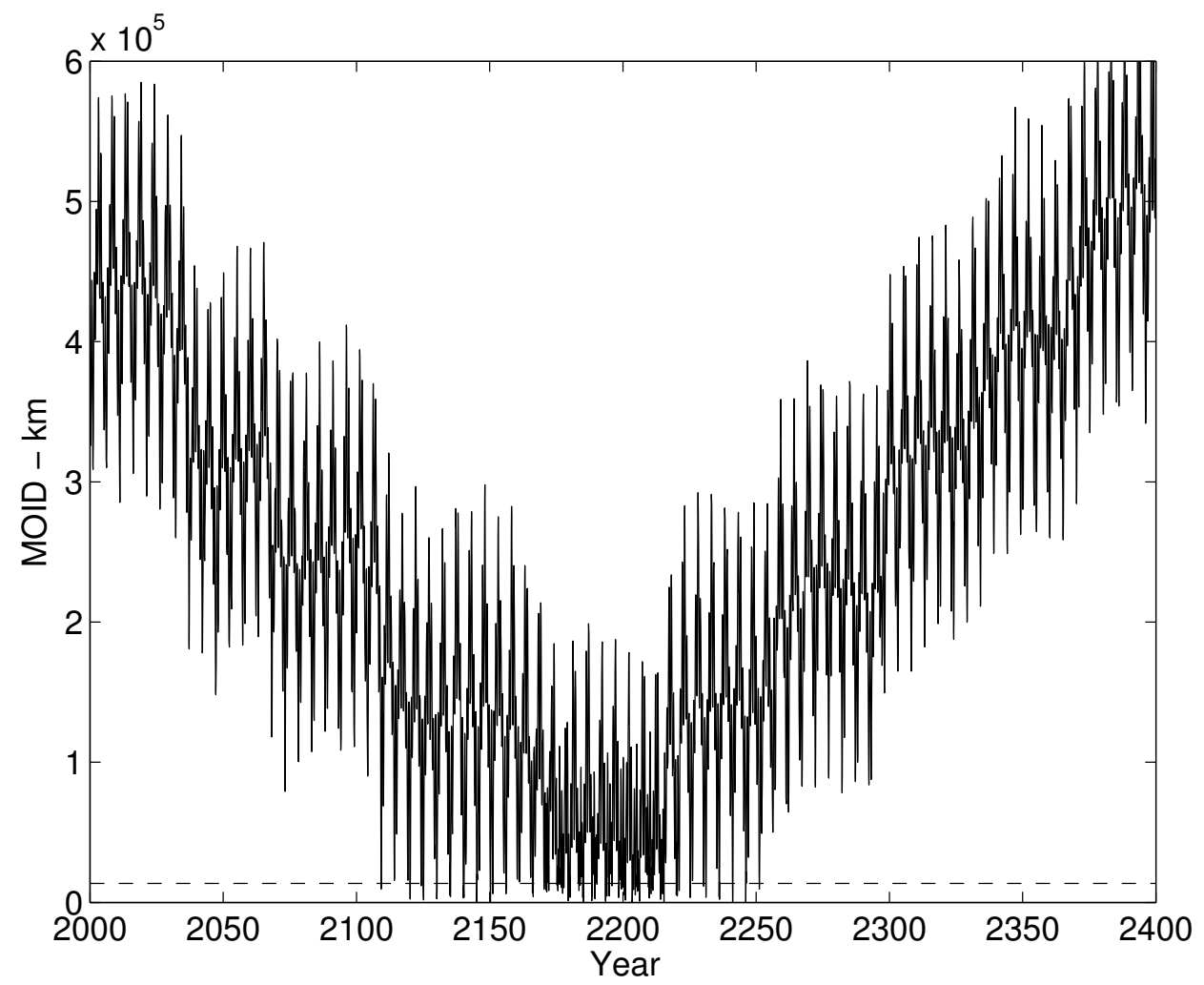

Fig. 11. - Time history of the MOID. The Earth impact cross-section (2.1 Earth radii) is marked by the dashed line. 


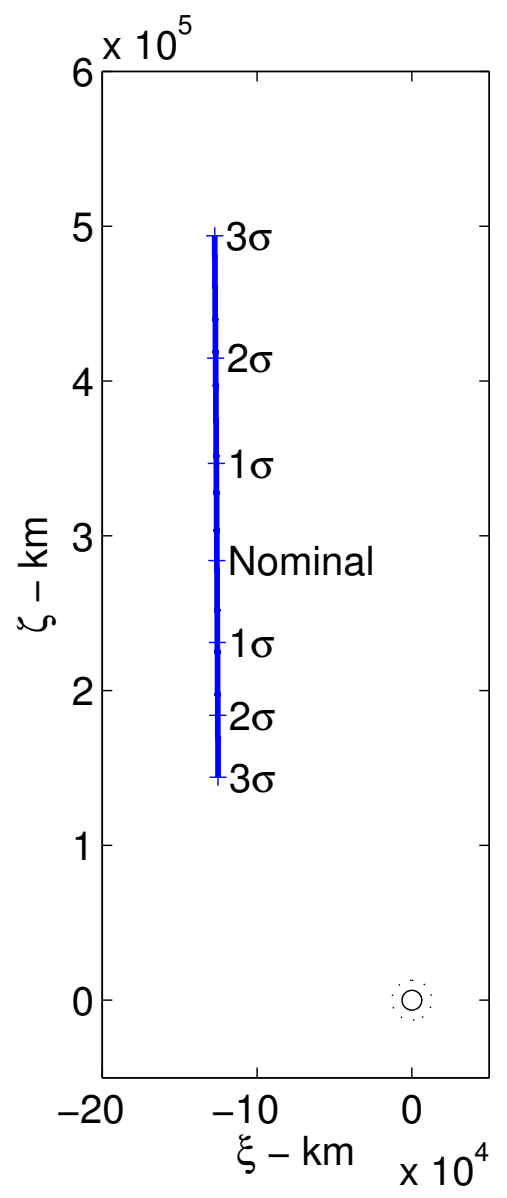

Fig. 12.- Uncertainty region on the $b$-plane of the 2135 encounter. The Earth is plotted to scale at the origin, and the impact cross-section is indicated by the dots. 


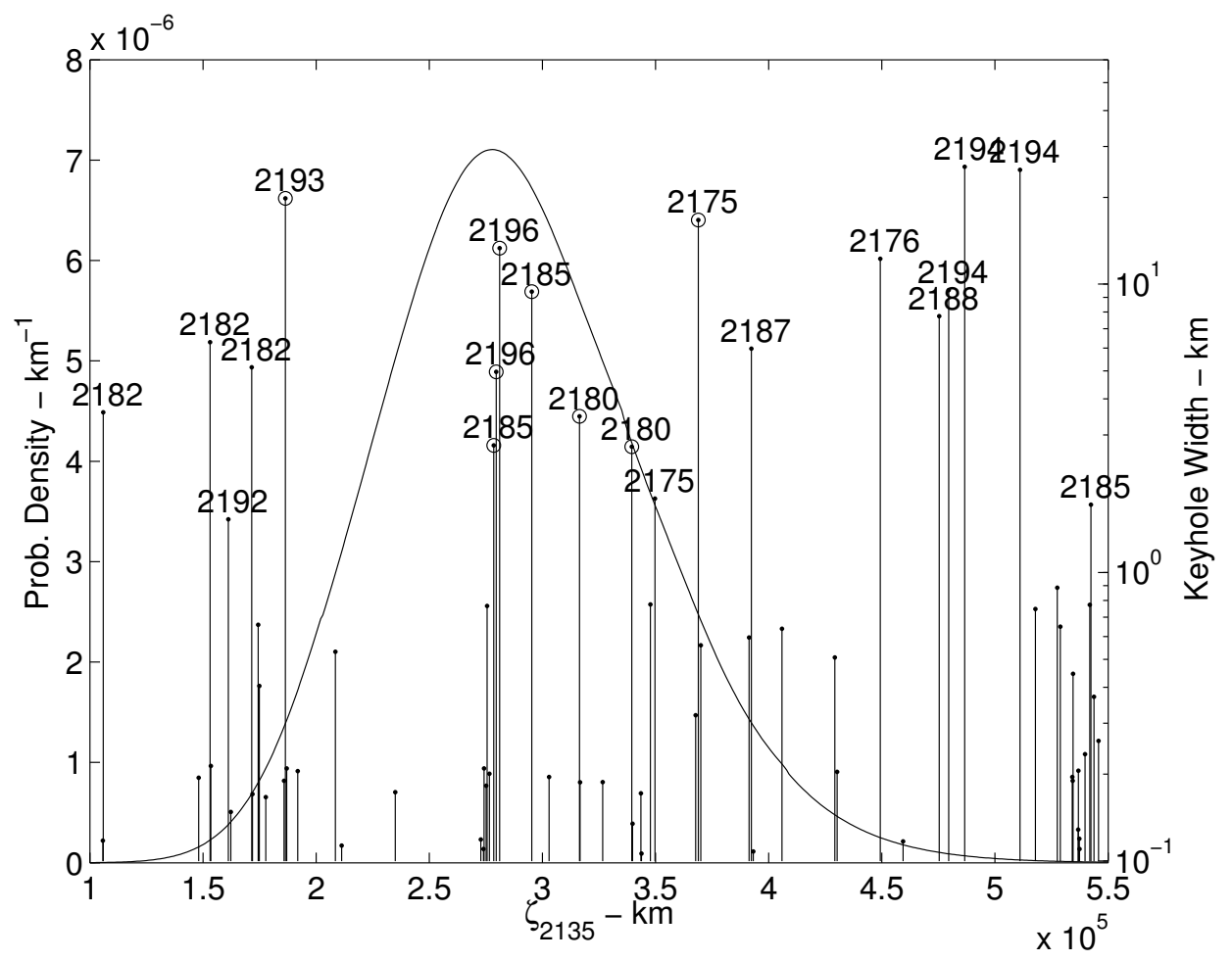

Fig. 13.- A map of the Bennu impact keyholes on the $2135 b$-plane. The probability density is given by the curve with the left ordinate, and the keyholes are indicated by the vertical lines at their respective $\zeta_{2135}$ positions with their widths given on the right ordinate. For clarity only keyholes wider than $1 \mathrm{~km}$ are labeled with the year of impact and only keyholes $>100 \mathrm{~m}$ in width are depicted. Potential impacts with impact probability greater than $10^{-5}$ are marked with a circle at the top of the vertical bar. 

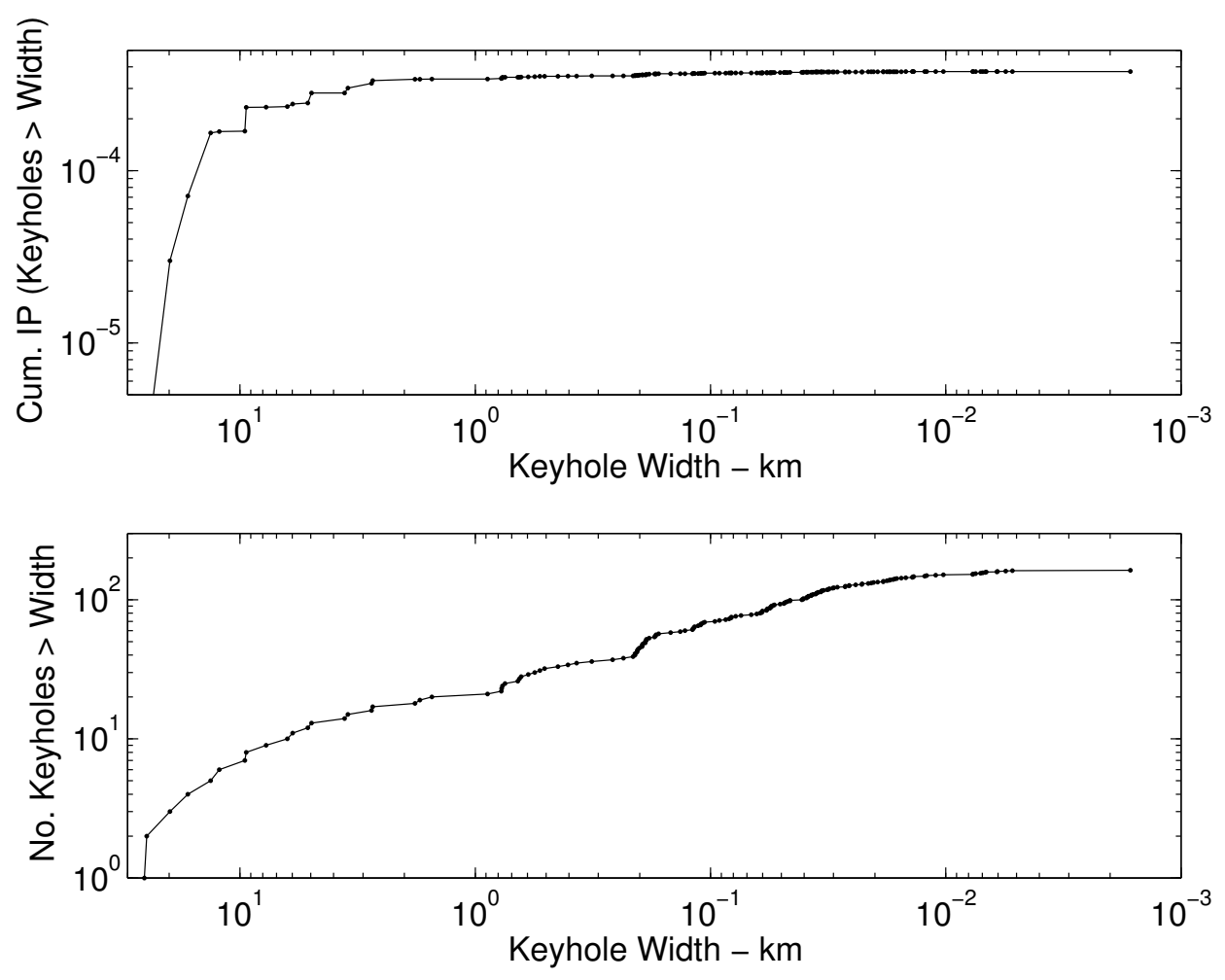

Fig. 14. - Cumulative impact probability (upper) and cumulative number of keyholes found (lower), each as a function of diminishing keyhole width. The cumulative impact probability over all potential impacts is $3.7 \times 10^{-4}$. 


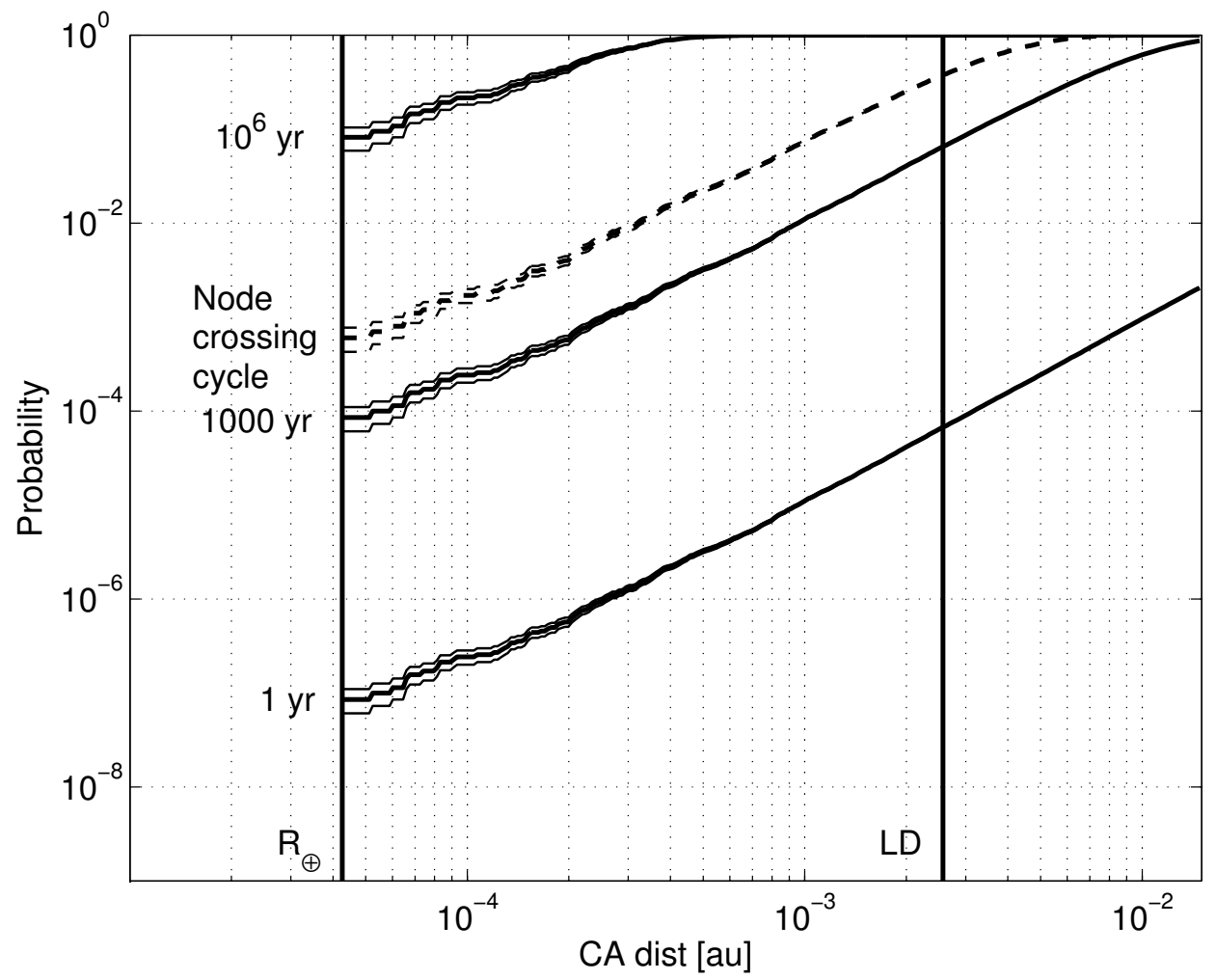

Fig. 15.- Probability (with corresponding $1 \sigma$ error bars) of having at least one close approach (CA) within a given distance for different time intervals. The dashed curve is for a node crossing cycle, while solid line are for $1 \mathrm{yr}, 1000 \mathrm{yr}$, and 1,000,000 yr. The Earth radius $\left(R_{\oplus}\right)$ and lunar distance (LD) are marked by vertical lines. 


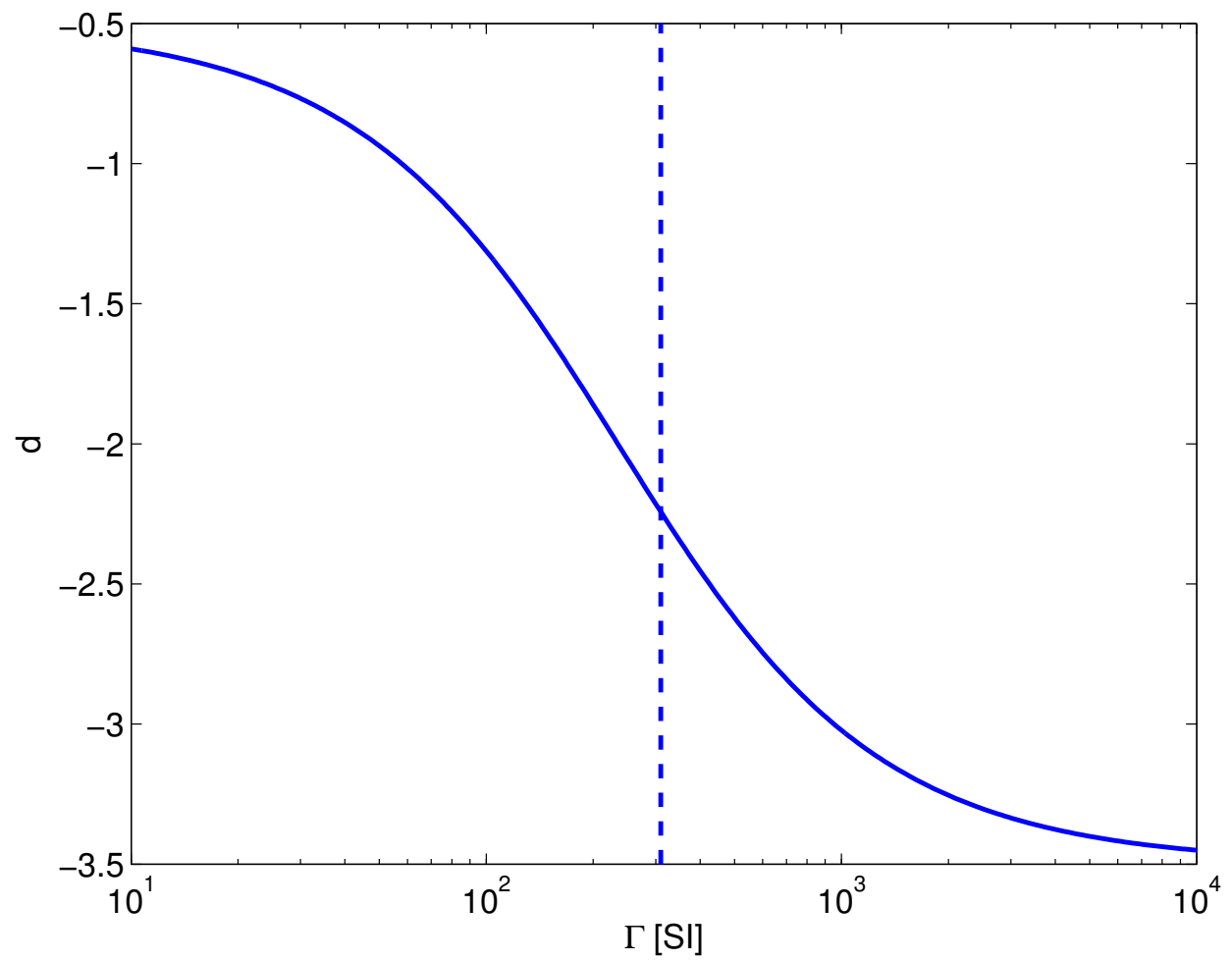

Fig. 16.- Dependency of transverse acceleration exponent $d$ on thermal inertia $\Gamma$ for Bennu. 


\section{List of Tables}

1 Radar Astrometry for $(101955)$ Bennu. . . . . . . . . . . . . . . . . 50

2 Radar observations of Bennu from 2011. Each line gives the UTC date and start/stop times, the number of transmit-receive cycles (runs), and the direction and distance to Bennu at the mid-epoch on each date. All observations used a 2 microsecond baud (corresponding to a range resolution of 300 meters), an 8191-length code, and JPL/Horizons orbital solution 70. . . . . . . . . . . 51

3 JPL orbit Solns. 85 and 87 for Bennu, Ecliptic J2000 Frame. . . . . . . . . . . 52

4 Physical characteristics of (101955) Bennu and associated marginal uncertainty in estimate of bulk density $\rho$. Tabulated error bars represent assumed $1 \sigma$ uncertainties. 53

5 Dynamical Effect of Several Model Variations. The columns indicate the type of model variation and the associated change in semimajor axis drift rate $d a / d t$ or $2135 b$-plane coordinates $\left(\xi_{2135}, \zeta_{2135}\right)$. Tabulated $\Delta$ values are with respect to JPL solution 87 (Table 3), for which $d a / d t=-18.973 \times 10^{-4} \mathrm{au} / \mathrm{My}$ at epoch 2011-Jan1.0 and $\left(\xi_{2135}, \zeta_{2135}\right)=(-125932 \mathrm{~km}, 281597 \mathrm{~km}) . \ldots \ldots \ldots \ldots$

6 Main belt asteroid perturbers, associated $G M$ values and the dynamical relevance of each as in the previous table. . . . . . . . . . . . . . 555

7 Bennu bulk density and related quantities with $1 \sigma$ uncertainties. . . . . . . . . 56

8 Bennu Deterministic Earth Approaches Closer than 0.05 AU (JPL solution 76). . . . 57

$9 \quad$ Keyholes in $2135 b$-plane and associated impact probabilities, JPL solution 76 . . . 58

10 Resonances associated with the eight potential impacts with impact probability $>10^{-5}$. [59]

11 Formal uncertainties with and without simulated OSIRIS-REx pseudo-range measurements as described in the text. . . . . . . . . . . . . . 60 
Table 1: Radar Astrometry for (101955) Bennu.

\begin{tabular}{|c|c|c|c|}
\hline $\begin{array}{l}\text { Date \& Time } \\
\text { (UTC - Receive) }\end{array}$ & $\begin{array}{r}\text { Measurement } \\
(\mu \mathrm{sec} \text { or } \mathrm{Hz})\end{array}$ & $\begin{array}{l}\text { Uncertainty } \\
(\mu \text { sec or Hz) }\end{array}$ & Station \\
\hline \multicolumn{4}{|l|}{ Delay } \\
\hline 2011-09-29 11:55:00 & 202378520.04 & 2.0 & Arecibo \\
\hline 2011-09-28 11:08:00 & 199711477.27 & 2.0 & Arecibo \\
\hline 2011-09-27 11:39:00 & 197293588.79 & 2.0 & Arecibo \\
\hline 2005-10-02 14:10:00 & 57762582.67 & 0.5 & Arecibo \\
\hline 2005-10-02 12:55:00 & 57594268.2 & 0.5 & Arecibo \\
\hline $2005-09-28$ 13:35:00 & 45734943.4 & 0.5 & Arecibo \\
\hline 2005-09-28 11:57:00 & 45550976.4 & 0.5 & Arecibo \\
\hline 2005-09-20 11:24:00 & 33024222.68 & 0.5 & Arecibo \\
\hline 2005-09-20 09:09:00 & 33024251.3 & 0.5 & Arecibo \\
\hline 2005-09-19 12:50:00 & 33215505.86 & 1.0 & Goldstone \\
\hline 2005-09-18 12:20:00 & 33873463 . & 1.0 & Goldstone \\
\hline 2005-09-16 09:27:00 & 36609699.09 & 0.5 & Arecibo \\
\hline 2005-09-16 08:45:00 & 36658796.03 & 0.5 & Arecibo \\
\hline 1999-10-01 13:40:00 & 35441297 . & 1.0 & Goldstone \\
\hline 1999-09-25 12:55:00 & 17785960.83 & 1.0 & Arecibo \\
\hline 1999-09-25 11:09:00 & 17634668.28 & 1.0 & Arecibo \\
\hline 1999-09-24 12:23:00 & 15955075.55 & 1.0 & Arecibo \\
\hline 1999-09-24 10:26:00 & 15838961.63 & 1.0 & Arecibo \\
\hline 1999-09-23 11:28:00 & 14846130.16 & 1.0 & Arecibo \\
\hline 1999-09-23 09:36:00 & 14800106.19 & 1.0 & Arecibo \\
\hline 1999-09-23 09:30:00 & 14820631. & 5.0 & Goldstone \\
\hline 1999-09-21 10:20:00 & 15418454 . & 10.0 & Goldstone \\
\hline \multicolumn{4}{|l|}{ Doppler } \\
\hline 2011-09-29 11:55:00 & -72841.0156 & 1.0 & Arecibo \\
\hline 2011-09-28 11:08:00 & -68554.7858 & 1.0 & Arecibo \\
\hline 2011-09-27 11:39:00 & -66400.2088 & 1.0 & Arecibo \\
\hline 2005-09-28 12:00:00 & -73137.0697 & 1.0 & Arecibo \\
\hline 2005-09-20 09:06:00 & 2631.7168 & 1.0 & Arecibo \\
\hline 2005-09-16 08:44:00 & 47170.7359 & 1.0 & Arecibo \\
\hline 1999-09-21 09:00:00 & 135959. & 5.0 & Goldstone \\
\hline
\end{tabular}

Notes:

- Transmit frequency is $2.38 \mathrm{GHz}$ at Arecibo and $8.56 \mathrm{GHz}$ at Goldstone.

- All measurements are referenced to the body center of mass.

- Measurements are also available online at http://ssd.jpl.nasa.gov/?radar. 
Table 2: Radar observations of Bennu from 2011. Each line gives the UTC date and start/stop times, the number of transmit-receive cycles (runs), and the direction and distance to Bennu at the mid-epoch on each date. All observations used a 2 microsecond baud (corresponding to a range resolution of 300 meters), an 8191-length code, and JPL/Horizons orbital solution 70.

\begin{tabular}{ccccccc}
\hline UTC Date & Start Time & Stop Time & Runs & RA $\left(^{\circ}\right)$ & DEC $\left(^{\circ}\right)$ & Distance $(\mathrm{au})$ \\
\hline 2011-Sep-27 & $10: 30: 36$ & $12: 53: 45$ & 21 & 114.3 & +28.3 & 0.1977 \\
2011-Sep-28 & $10: 34: 40$ & $12: 56: 31$ & 10 & 116.2 & +28.5 & 0.2002 \\
2011-Sep-29 & $10: 38: 31$ & $13: 02: 29$ & 18 & 118.1 & +28.6 & 0.2028 \\
\hline
\end{tabular}


Table 3: JPL orbit Solns. 85 and 87 for Bennu, Ecliptic J2000 Frame.

\begin{tabular}{ll}
\hline Solution 85 (Nonlinear Yarkovsky Model) \\
Epoch & 2011-Jan-1.0 TDB \\
Semimajor axis $(a)$ & $1.126391025996(42) \mathrm{au}$ \\
Eccentricity $(e)$ & $0.203745112(21)$ \\
Perihelion dist. $(q)$ & $0.896894360(24)$ au \\
Perihelion time $\left(t_{p}\right)$ & $2010-$ Aug-30.6419463(30) TDB \\
Long. of Asc. Node $(\Omega)$ & $2.0608668(37)^{\circ}$ \\
Arg. of perihelion $(\omega)$ & $66.2230699(55)^{\circ}$ \\
Inclination $(i)$ & $6.0349391(27)^{\circ}$ \\
Bulk Density $(\rho)$ & $1181.1(6.3) \mathrm{kg} / \mathrm{m}^{3} \dagger$ \\
$\chi^{2} \ddagger$ & 68.37 \\
& \\
Solution 87 $($ Transverse & Yarkovsky Model, $d=2.25)$ \\
Epoch & $2011-J a n-1.0 \mathrm{TDB}$ \\
Semimajor axis $(a)$ & $1.126391026404(40) \mathrm{au}$ \\
Eccentricity $(e)$ & $0.203745114(21)$ \\
Perihelion dist. $(q)$ & $0.896894358(24)$ au \\
Perihelion time $\left(t_{p}\right)$ & $2010-$ Aug-30.6419468 $(30)$ TDB \\
Long. of Asc. Node $(\Omega)$ & $2.0608670(37)^{\circ}$ \\
Arg. of perihelion $(\omega)$ & $66.2230705(55)^{\circ}$ \\
Inclination $(i)$ & $6.0349388(27)^{\circ}$ \\
Transverse accel. $\left(A_{T}\right)$ & $-4.618(24) \times 10^{-14}$ au/d ${ }^{2}$ \\
$\chi^{2} \ddagger$ & 68.73 \\
\hline
\end{tabular}

Notes:

Numbers in parentheses indicate the $1 \sigma$ formal uncertainties of the corresponding (last two) digits in the parameter value.

$\dagger$ The bulk density uncertainty is marginal only with respect to the orbital elements, and is conditional with respect to the physical parameters that can affect the thermal modeling. In particular, the uncertainties in effective diameter, thermal inertia and obliquity are not captured here, and these lead to a marginal uncertainty an order of magnitude greater. See Fig. 9 and the discussion in Sec. 4. $\ddagger \chi^{2}$ denotes the sum of squares of normalized postfit residuals. 
Table 4: Physical characteristics of (101955) Bennu and associated marginal uncertainty in estimate of bulk density $\rho$. Tabulated error bars represent assumed $1 \sigma$ uncertainties.

\begin{tabular}{llll}
\hline Parameter & Value \& Uncertainty & Ref. & $\rho$ Uncert. \\
\hline Thermal inertia & $\Gamma=310 \pm 70 \mathrm{~J} \mathrm{~m}^{-1} \mathrm{~s}^{-0.5} \mathrm{~K}^{-1}$ & $\mathrm{~A}$ & $+2.1 \% /-4.1 \%$ \\
Cross-sectional Area/Volume & $A / V=3.06 \pm 0.06 \times 10^{-3} \mathrm{~m}^{-1}$ & $\mathrm{~B}$ & $\pm 2.0 \%$ \\
Obliquity of equator & $\gamma=175^{\circ} \pm 4^{\circ}$ & $\mathrm{B}$ & $+0.4 \% /-0.9 \%$ \\
Surface emissivity & $\epsilon=0.90 \pm 0.05$ & $\mathrm{~A}$ & $\pm 0.3 \%$ \\
Bond albedo & $A=0.017 \pm 0.002$ & $\mathrm{~A}$ & $\pm 0.2 \%$ \\
Rotation period & $4.29746 \pm 0.002 \mathrm{~h}$ & $\mathrm{~B}$ & $\pm 0.0 \%$ \\
\hline
\end{tabular}

References: A-Emery et al. (2014), B- Nolan et al. (2013) 
Table 5: Dynamical Effect of Several Model Variations. The columns indicate the type of model variation and the associated change in semimajor axis drift rate $d a / d t$ or $2135 b$-plane coordinates $\left(\xi_{2135}, \zeta_{2135}\right)$. Tabulated $\Delta$ values are with respect to JPL solution 87 (Table 3), for which $d a / d t=$ $-18.973 \times 10^{-4} \mathrm{au} / \mathrm{My}$ at epoch 2011-Jan-1.0 and $\left(\xi_{2135}, \zeta_{2135}\right)=(-125932 \mathrm{~km}, 281597 \mathrm{~km})$.

\begin{tabular}{|c|c|c|c|c|}
\hline Model & $\begin{array}{c}\Delta d a / d t \\
\left(10^{-4} \mathrm{au} / \mathrm{My}\right)\end{array}$ & $\begin{array}{r}\Delta \xi_{2135} \\
(\mathrm{~km})\end{array}$ & $\begin{array}{r}\Delta \zeta_{2135} \\
(\mathrm{~km})\end{array}$ & Remarks \\
\hline \multicolumn{5}{|l|}{ Yarkovsky Model } \\
\hline Nonlinear & -0.004 & -43 & 6251 & Soln. 85 \\
\hline Linear & 0.006 & -16 & 2096 & Soln. 86 \\
\hline$d=2.25$ & 0.000 & 0 & 0 & Soln. 87 \\
\hline$d=2.00$ & 0.006 & -21 & 3423 & \\
\hline \multicolumn{5}{|c|}{ Asteroid Perturbations } \\
\hline 25 Perturbers & 0.000 & 0 & 0 & Soln. 87 \\
\hline BIG-16 only & -0.004 & 2 & -409 & \\
\hline CPVH only & -0.010 & 22 & -3714 & \\
\hline \multicolumn{5}{|c|}{ Earth Oblateness Limit } \\
\hline 10. au & 0.000 & 0 & -70 & \\
\hline 1. au & 0.000 & 0 & 0 & Soln. 87 \\
\hline $0.1 \mathrm{au}$ & 0.000 & 0 & -25 & \\
\hline $0.01 \mathrm{au}$ & -0.004 & 11 & -1822 & \\
\hline $0.001 \mathrm{au}$ & -0.004 & 10 & -1703 & \\
\hline \multicolumn{5}{|l|}{ Relativity Model } \\
\hline Full EIH & 0.000 & 0 & 0 & Soln. 87 \\
\hline Basic Sun Model & 0.305 & -1128 & 168469 & \\
\hline EIH Sun only & 0.295 & -1069 & 160086 & \\
\hline w/o Mercury & -0.001 & 1 & -240 & \\
\hline w/o Venus & 0.017 & -54 & 8954 & \\
\hline w/o Earth & 0.291 & -1059 & 159130 & \\
\hline w/o Mars & 0.000 & 0 & -43 & \\
\hline w/o Jupiter & -0.012 & 46 & -7652 & \\
\hline w/o Saturn & -0.004 & 11 & -1859 & \\
\hline w/o Uranus & 0.000 & -1 & 168 & \\
\hline w/o Neptune & 0.000 & -0 & 60 & \\
\hline w/o Moon & 0.004 & -11 & 1801 & \\
\hline \multicolumn{5}{|l|}{ Outlier Rejection } \\
\hline$\chi_{\mathrm{rej}}=3$ & 0.049 & -537 & 84670 & 7 del. \\
\hline$\chi_{\mathrm{rej}}=2$ & 0.026 & -414 & 65916 & 15 del. \\
\hline$\chi_{\mathrm{rej}}=1.5$ & 0.018 & -243 & 39502 & 24 del. \\
\hline$\chi_{\mathrm{rej}}=1$ & 0.003 & -13 & 2220 & 49 del. \\
\hline Manual & 0.000 & 0 & 0 & 91 del., Soln. 87 \\
\hline \multicolumn{5}{|l|}{ Integration Tolerance } \\
\hline $10^{-16}$ & 0.000 & -0 & 60 & \\
\hline $10^{-15}$ & 0.000 & 0 & 0 & Soln. 87 \\
\hline $10^{-14}$ & -0.002 & 4 & -721 & \\
\hline $10^{-13}$ & -0.003 & -5 & 831 & \\
\hline \multicolumn{5}{|l|}{ Other } \\
\hline Area $/$ Mass $=0$ & -0.001 & 5 & -1122 & \\
\hline DE405 w/BIG-16 & -0.048 & 84 & -14160 & \\
\hline
\end{tabular}

Note: For reference, the formal uncertainty in the $d a / d t$ estimate is $0.100 \times 10^{-4}$ au/My and in $\zeta_{2135}$ is roughly $60,000 \mathrm{~km}$. 
Table 6: Main belt asteroid perturbers, associated $G M$ values and the dynamical relevance of each as in the previous table.

\begin{tabular}{|c|c|c|c|c|c|c|}
\hline Count & $\begin{array}{l}\text { IAU } \\
\text { No. }\end{array}$ & Name & $\begin{array}{c}G M \\
\left(\mathrm{~km}^{3} / \mathrm{s}^{2}\right)\end{array}$ & $\begin{array}{c}\Delta d a / d t \\
\left(10^{-4} \mathrm{au} / \mathrm{My}\right)\end{array}$ & $\begin{array}{c}\Delta \xi_{2135} \\
(\mathrm{~km})\end{array}$ & $\begin{array}{c}\Delta \zeta_{2135} \\
(\mathrm{~km})\end{array}$ \\
\hline \multicolumn{7}{|l|}{$\mathrm{CPVH}$} \\
\hline 1 & 1 & Ceres & $63.13^{a}$ & -0.118 & 298 & -51351 \\
\hline 2 & 2 & Pallas & $13.73^{b}$ & -0.109 & 295 & -50754 \\
\hline 3 & 4 & Vesta & $17.29^{c}$ & 0.437 & -893 & 136194 \\
\hline 4 & 10 & Hygiea & $5.78^{a}$ & 0.002 & -11 & 1661 \\
\hline \multicolumn{7}{|c|}{ Additions for BIG-16 } \\
\hline 5 & 3 & Juno & $1.82^{d}$ & 0.002 & -8 & 1126 \\
\hline 6 & 6 & Hebe & $0.93^{d}$ & 0.019 & -65 & 10790 \\
\hline 7 & 7 & Iris & $0.86^{d}$ & -0.014 & 57 & -9532 \\
\hline 8 & 15 & Eunomia & $2.10^{d}$ & -0.001 & 1 & -260 \\
\hline 9 & 16 & Psyche & $1.81^{d}$ & -0.005 & 16 & -2615 \\
\hline 10 & 29 & Amphitrite & $0.86^{d}$ & 0.000 & -0 & 30 \\
\hline 11 & 52 & Europa & $1.59^{d}$ & 0.001 & -5 & 643 \\
\hline 12 & 65 & Cybele & $0.91^{d}$ & 0.000 & 1 & -188 \\
\hline 13 & 87 & Sylvia & $0.99^{d}$ & 0.000 & -1 & 66 \\
\hline 14 & 88 & Thisbe & $1.02^{d}$ & -0.007 & 19 & -3099 \\
\hline 15 & 511 & Davida & $2.26^{d}$ & 0.000 & 1 & -114 \\
\hline 16 & 704 & Interamnia & $2.19^{d}$ & 0.000 & 0 & -7 \\
\hline \multicolumn{7}{|c|}{ Additions for Bennu } \\
\hline 17 & 11 & Parthenope & $0.39^{d}$ & -0.006 & 18 & -3031 \\
\hline 18 & 14 & Irene & $0.19^{d}$ & -0.002 & 6 & -1013 \\
\hline 19 & 56 & Melete & $0.31^{d}$ & -0.002 & -6 & 1070 \\
\hline 20 & 63 & Ausonia & $0.10^{d}$ & -0.002 & 4 & -731 \\
\hline 21 & 135 & Hertha & $0.08^{d}$ & 0.001 & -6 & 872 \\
\hline 22 & 259 & Aletheia & $0.52^{d}$ & 0.000 & 0 & -31 \\
\hline 23 & 324 & Bamberga & $0.69^{d}$ & -0.001 & -4 & 628 \\
\hline 24 & 419 & Aurelia & $0.12^{d}$ & 0.002 & 2 & -259 \\
\hline 25 & 532 & Herculina & $0.77^{d}$ & 0.005 & -13 & 2200 \\
\hline
\end{tabular}

Refs.: ${ }^{d}$ Baer et al. (2011), ${ }^{\text {Konopliv et al. }}$ (2011), ${ }^{\text {Russell et al. }}$ (2012), ${ }^{d}$ Carry $(2012)$. 
Table 7: Bennu bulk density and related quantities with $1 \sigma$ uncertainties.

\begin{tabular}{ll}
\hline Bulk density $\rho\left(\mathrm{kg} / \mathrm{m}^{3}\right)$ & $1260 \pm 70$ \\
Mass $M\left(10^{10} \mathrm{~kg}\right)$ & $7.8 \pm 0.9$ \\
$G M\left(\mathrm{~m}^{3} / \mathrm{s}^{2}\right)$ & $5.2 \pm 0.6$ \\
Area-to-mass ratio $\left(10^{-6} \mathrm{~m}^{2} / \mathrm{kg}\right)$ & $2.4 \pm 0.1$ \\
\hline
\end{tabular}


Table 8: Bennu Deterministic Earth Approaches Closer than 0.05 AU (JPL solution 76).

\begin{tabular}{ccccrr}
\hline Date (TDB) & $\begin{array}{c}\text { Nom. Dist. } \\
(\mathrm{AU})\end{array}$ & $\begin{array}{c}3 \sigma \text { Min. } \\
(\mathrm{AU})\end{array}$ & $\begin{array}{c}3 \sigma \text { Max. } \\
(\mathrm{AU})\end{array}$ & $\begin{array}{r}1 \sigma \text { Time Uncert. } \\
(\mathrm{s})\end{array}$ & $\begin{array}{r}1 \sigma \zeta \text { Uncert. } \\
(\mathrm{km})\end{array}$ \\
\hline 1654 Sep 17.89194 & 0.022033 & 0.010586 & 0.035240 & 49590 & 816253 \\
1788 Sep 20.56364 & 0.009771 & 0.009304 & 0.010254 & 2237 & 32739 \\
1848 Sep 21.91904 & 0.007915 & 0.007892 & 0.007938 & 105 & 1573 \\
1911 Sep 22.88762 & 0.014178 & 0.014177 & 0.014179 & 2.4 & 38 \\
1970 Sep 27.10790 & 0.021403 & 0.021403 & 0.021403 & 1.6 & 16 \\
1999 Sep 22.76422 & 0.014686 & 0.014686 & 0.014686 & $\ll 1$ & 1.1 \\
2005 Sep 20.44528 & 0.033130 & 0.033130 & 0.033130 & $\ll 1$ & 1.2 \\
2054 Sep 30.04163 & 0.039299 & 0.039299 & 0.039299 & 1.6 & 11 \\
2060 Sep 23.02530 & 0.005008 & 0.005008 & 0.005008 & 1.0 & 15 \\
2080 Sep 22.02378 & 0.015560 & 0.015427 & 0.015693 & 360 & 7318 \\
2135 Sep 25.40942 & 0.002009 & 0.000819 & 0.003549 & 4746 & 79674 \\
\hline
\end{tabular}


Table 9: Keyholes in 2135 b-plane and associated impact probabilities, JPL solution 76.

\begin{tabular}{|c|c|c|c|c|c|c|c|c|c|}
\hline Year & $\begin{array}{l}\zeta_{2135} \\
(\mathrm{~km})\end{array}$ & $\begin{array}{r}\zeta \text {-width } \\
(\mathrm{km})\end{array}$ & $\begin{array}{c}\text { Impact } \\
\text { Prob. }\end{array}$ & $\begin{array}{c}\text { Palermo } \\
\text { Scale }\end{array}$ & Year & $\begin{array}{l}\zeta_{2135} \\
(\mathrm{~km})\end{array}$ & $\begin{array}{r}\zeta \text {-width } \\
(\mathrm{km})\end{array}$ & $\begin{array}{l}\text { Impact } \\
\text { Prob. }\end{array}$ & $\begin{array}{c}\text { Palermo } \\
\text { Scale }\end{array}$ \\
\hline 2192 & 92981 & 0.17 & $1.04 \times 10^{-10}$ & -8.27 & 2195 & 343783 & 0.11 & $4.15 \times 10^{-07}$ & -4.67 \\
\hline 2187 & 93280 & 0.32 & $2.01 \times 10^{-10}$ & -7.97 & 2181 & 347699 & 0.78 & $2.86 \times 10^{-06}$ & -3.80 \\
\hline 2198 & 99162 & 0.25 & $3.33 \times 10^{-10}$ & -7.78 & 2175 & 349704 & 1.80 & $6.43 \times 10^{-06}$ & -3.43 \\
\hline 2187 & 105731 & 0.12 & $3.44 \times 10^{-10}$ & -7.74 & 2186 & 367729 & 0.32 & $8.12 \times 10^{-07}$ & -4.36 \\
\hline 2182 & 105945 & 3.59 & $1.08 \times 10^{-08}$ & -6.23 & 2175 & 368877 & 16.67 & $4.13 \times 10^{-05}$ & -2.63 \\
\hline 2197 & 148127 & 0.19 & $3.08 \times 10^{-08}$ & -5.81 & 2181 & 370007 & 0.56 & $1.35 \times 10^{-06}$ & -4.13 \\
\hline 2182 & 153125 & 6.29 & $1.42 \times 10^{-06}$ & -4.11 & 2193 & 391326 & 0.60 & $8.52 \times 10^{-07}$ & -4.36 \\
\hline 2187 & 153481 & 0.21 & $4.94 \times 10^{-08}$ & -5.58 & 2187 & 392308 & 5.97 & $8.34 \times 10^{-06}$ & -3.35 \\
\hline 2192 & 161203 & 1.53 & $5.83 \times 10^{-07}$ & -4.52 & 2198 & 393200 & 0.11 & $1.47 \times 10^{-07}$ & -5.13 \\
\hline 2193 & 162322 & 0.15 & $6.04 \times 10^{-08}$ & -5.51 & 2181 & 405893 & 0.64 & $6.27 \times 10^{-07}$ & -4.46 \\
\hline 2182 & 171554 & 5.15 & $3.55 \times 10^{-06}$ & -3.71 & 2187 & 429187 & 0.51 & $2.43 \times 10^{-07}$ & -4.89 \\
\hline 2187 & 171842 & 0.17 & $1.19 \times 10^{-07}$ & -5.20 & 2181 & 430234 & 0.20 & $9.41 \times 10^{-08}$ & -5.28 \\
\hline 2187 & 174357 & 0.66 & $5.25 \times 10^{-07}$ & -4.55 & 2176 & 449272 & 12.23 & $3.07 \times 10^{-06}$ & -3.76 \\
\hline 2192 & 174935 & 0.40 & $3.31 \times 10^{-07}$ & -4.76 & 2193 & 459392 & 0.12 & $2.09 \times 10^{-08}$ & -5.97 \\
\hline 2194 & 177766 & 0.17 & $1.57 \times 10^{-07}$ & -5.09 & 2188 & 475391 & 7.74 & $7.99 \times 10^{-07}$ & -4.37 \\
\hline 2199 & 185793 & 0.19 & $2.56 \times 10^{-07}$ & -4.89 & 2194 & 479545 & 9.54 & $8.50 \times 10^{-07}$ & -4.36 \\
\hline 2193 & 186415 & 19.84 & $2.75 \times 10^{-05}$ & -2.85 & 2194 & 486578 & 25.51 & $1.77 \times 10^{-06}$ & -4.04 \\
\hline 2198 & 186996 & 0.21 & $2.97 \times 10^{-07}$ & -4.83 & 2194 & 511097 & 24.90 & $6.94 \times 10^{-07}$ & -4.45 \\
\hline 2199 & 191883 & 0.20 & $3.53 \times 10^{-07}$ & -4.75 & 2188 & 517881 & 0.75 & $1.61 \times 10^{-08}$ & -6.07 \\
\hline 2198 & 208524 & 0.53 & $1.53 \times 10^{-06}$ & -4.11 & 2193 & 527510 & 0.89 & $1.30 \times 10^{-08}$ & -6.17 \\
\hline 2190 & 211276 & 0.11 & $3.50 \times 10^{-07}$ & -4.74 & 2181 & 528840 & 0.65 & $9.06 \times 10^{-09}$ & -6.30 \\
\hline 2199 & 235014 & 0.17 & $8.69 \times 10^{-07}$ & -4.36 & 2193 & 534125 & 0.20 & $2.21 \times 10^{-09}$ & -6.94 \\
\hline 2192 & 272823 & 0.12 & $8.39 \times 10^{-07}$ & -4.36 & 2193 & 534310 & 0.19 & $2.13 \times 10^{-09}$ & -6.96 \\
\hline 2198 & 273950 & 0.11 & $7.79 \times 10^{-07}$ & -4.41 & 2198 & 534527 & 0.45 & $4.96 \times 10^{-09}$ & -6.60 \\
\hline 2186 & 274243 & 0.21 & $1.48 \times 10^{-06}$ & -4.10 & 2198 & 536801 & 0.13 & $1.30 \times 10^{-09}$ & -7.18 \\
\hline 2191 & 275096 & 0.18 & $1.29 \times 10^{-06}$ & -4.17 & 2198 & 536823 & 0.21 & $2.08 \times 10^{-09}$ & -6.98 \\
\hline 2191 & 275569 & 0.77 & $5.44 \times 10^{-06}$ & -3.55 & 2196 & 537259 & 0.12 & $1.19 \times 10^{-09}$ & -7.22 \\
\hline 2191 & 276541 & 0.20 & $1.42 \times 10^{-06}$ & -4.13 & 2189 & 537324 & 0.11 & $1.09 \times 10^{-09}$ & -7.24 \\
\hline 2185 & 278479 & 2.76 & $1.96 \times 10^{-05}$ & -2.98 & 2199 & 539780 & 0.23 & $2.11 \times 10^{-09}$ & -6.98 \\
\hline 2196 & 279590 & 4.96 & $3.52 \times 10^{-05}$ & -2.75 & 2185 & 541921 & 0.77 & $6.37 \times 10^{-09}$ & -6.46 \\
\hline 2196 & 281070 & 13.32 & $9.45 \times 10^{-05}$ & -2.32 & 2185 & 542397 & 1.72 & $1.39 \times 10^{-08}$ & -6.12 \\
\hline 2185 & 295318 & 9.42 & $6.33 \times 10^{-05}$ & -2.47 & 2185 & 543753 & 0.37 & $2.84 \times 10^{-09}$ & -6.81 \\
\hline 2190 & 302940 & 0.20 & $1.24 \times 10^{-06}$ & -4.18 & 2197 & 545747 & 0.26 & $1.84 \times 10^{-09}$ & -7.03 \\
\hline 2180 & 316352 & 3.48 & $1.95 \times 10^{-05}$ & -2.96 & 2190 & 558169 & 11.57 & $4.86 \times 10^{-08}$ & -5.59 \\
\hline 2191 & 316680 & 0.19 & $1.05 \times 10^{-06}$ & -4.26 & 2196 & 561278 & 12.31 & $4.54 \times 10^{-08}$ & -5.64 \\
\hline 2186 & 326599 & 0.19 & $9.38 \times 10^{-07}$ & -4.30 & 2196 & 565865 & 20.20 & $6.12 \times 10^{-08}$ & -5.51 \\
\hline 2180 & 339506 & 2.73 & $1.14 \times 10^{-05}$ & -3.20 & 2190 & 618399 & 17.45 & $4.95 \times 10^{-09}$ & -6.59 \\
\hline 2191 & 339838 & 0.13 & $5.60 \times 10^{-07}$ & -4.53 & 2184 & 636299 & 54.23 & $6.51 \times 10^{-09}$ & -6.45 \\
\hline 2190 & 343541 & 0.17 & $6.74 \times 10^{-07}$ & -4.45 & 2184 & 651571 & 4.35 & $2.45 \times 10^{-10}$ & -7.88 \\
\hline
\end{tabular}

Note: Impact probabilities $>10^{-5}$ are highlighted in bold. 
Table 10: Resonances associated with the eight potential impacts with impact probability $>10^{-5}$.

\begin{tabular}{cccccc}
\hline Year & $\begin{array}{c}\zeta_{2135} \\
(\mathrm{~km})\end{array}$ & $\begin{array}{c}\text { Post-2135 Period } \\
(\mathrm{yr})\end{array}$ & Resonance & $\begin{array}{c}\text { Res. Period } \\
(\mathrm{yr})\end{array}$ & $\begin{array}{c}\Delta P \\
(\mathrm{yr})\end{array}$ \\
\hline 2193 & 186415 & 1.2342 & $58 \mathrm{yr} / 47 \mathrm{rev}$ & 1.2340 & +0.0002 \\
2185 & 278479 & 1.2215 & $50 \mathrm{yr} / 41 \mathrm{rev}$ & 1.2195 & +0.0020 \\
2196 & 279590 & 1.2213 & $61 \mathrm{yr} / 50 \mathrm{rev}$ & 1.2200 & +0.0013 \\
2196 & 281070 & 1.2211 & $61 \mathrm{yr} / 50 \mathrm{rev}$ & 1.2200 & +0.0011 \\
2185 & 295318 & 1.2194 & $50 \mathrm{yr} / 41 \mathrm{rev}$ & 1.2195 & -0.0001 \\
2180 & 316352 & 1.2169 & $45 \mathrm{yr} / 37 \mathrm{rev}$ & 1.2162 & +0.0007 \\
2180 & 339506 & 1.2144 & $45 \mathrm{yr} / 37 \mathrm{rev}$ & 1.2162 & -0.0018 \\
2175 & 368877 & 1.2116 & $40 \mathrm{yr} / 33 \mathrm{rev}$ & 1.2121 & -0.0005 \\
\hline
\end{tabular}


Table 11: Formal uncertainties with and without simulated OSIRIS-REx pseudo-range measurements as described in the text.

\begin{tabular}{lcc}
\hline & \multicolumn{2}{c}{ Uncertainties } \\
& Reference & With sim. obs. \\
\hline$A_{T}\left(10^{-16} \mathrm{au} / \mathrm{d}\right)$ & 2.52 & 0.38 \\
2020-Jan-04 Position $(\mathrm{km})$ & \\
$\quad 1.539$ & 0.008 \\
Radial & 0.855 & 0.033 \\
Transverse & 2.461 & 0.098 \\
$\quad$ Normal & & \\
2020-Jan-04 Velocity $(\mathrm{mm} / \mathrm{s})$ & \\
$\quad$ Radial & 0.662 & 0.005 \\
Transverse & 0.400 & 0.002 \\
$\quad$ Normal & 1.388 & 0.029 \\
$\zeta_{2135}(\mathrm{~km})$ & 58000 & 1000 \\
\hline
\end{tabular}

